"Long-term care policy with nonlinear strategic bequests"

Chiara Canta and Helmuth Cremer 


\title{
Long-term care policy with nonlinear strategic bequests*
}

\author{
Chiara Canta \\ Department of Economics, Finance and Law \\ Toulouse Business School \\ 31068 Toulouse, France \\ Helmuth Cremer \\ Toulouse School of Economics, \\ University of Toulouse Capitole \\ 31015 Toulouse, France
}

January 2017, revised Septembre 2017

*Financial support from the Chaire "Marché des risques et creation de valeur" of the FdR/SCOR is gratefully acknowledged. This paper has been presented at the EHEW 2017 in Oslo, the LAGV conference 2017 in Aix-en-Provence, and in seminars at the Universidad Autònoma de Barcelona and the Frisch Center in Oslo. We thank all the participants and particularly Marcos Vera-Hernández for their comments. 


\begin{abstract}
We study the design of long-term care (LTC) policy when children differ in their cost of providing informal care. Parents do not observe this cost, but they can commit to a "bequests rule" specifying a transfer conditional on the level of informal care. Care provided by high-cost children is distorted downwards in order to minimize the rent of low-cost ones. Social LTC insurance is designed to maximize a weighted sum of parents' and children's utility. The optimal uniform public LTC provision strikes a balance between insurance and children's utility. Under decreasing absolute risk aversion less than full insurance is provided to mitigate the distortion on informal care which reduces children's rents. A nonuniform policy conditioning LTC benefits on bequests provides full insurance even against the risk of having children with a high cost of providing care. Quite surprisingly the level of informal care induced by the optimal (uniform or nonuniform) policy always increases in the children's' welfare weight.
\end{abstract}

JEL classification: H2, H5, I13, J14.

Keywords: Long-term care, informal care, strategic bequests, asymmetric information. 


\section{Introduction}

Old age dependence and the need for long-term care (LTC) it brings about represents a major societal challenge in most developed countries. Due to population ageing the number of dependent elderly with cognitive and physical impairments will increase dramatically during the decades to come (Cremer et al., 2012). Dependence represents a significant financial risk of which only a small part is typically covered by social insurance. Private insurance markets are also thin. Instead, individuals rely on their savings or on informal care provided by family members. Currently the family is the main provider and informal care represents roughly $2 / 3$ of total care (Norton, 2000). Informal provision has no direct bearing on public finances, but it is not available to everyone. Whether this solidarity is sustainable at its current level is an important question. Sources of concerns are numerous. The drastic change in family values, the increasing number of childless households, the mobility of children, the increasing labor participation of women are as many factors explaining why the number of dependent elderly who cannot count on family solidarity, at least not for the full amount of care they need, is increasing. Furthermore, it is not clear if the important role played by informal care is desirable. Its real costs are often "hidden". In particular, it may indeed impose a significant burden on the caregivers which is both financial and psychological. ${ }^{1}$

In a nutshell, the current situation is inefficient as it leaves some elderly without proper care and often imposes a considerable burden on caregivers. This market failure creates a potential role of public intervention through social LTC provision or insurance. However, the public LTC policy will interact with informal care, and more generally with the exchanges within the family; consequently, policy design has to account for the induced changes in transfers within the family.

Informal care can be motivated by some form of altruism, result from implicit exchanges or be "imposed" by social norms. Knowing the foundation of informal care is

\footnotetext{
${ }^{1}$ On the costs borne by caregivers, see Colombo et al. (2011) or Coe and Van Houtven (2009).
} 
very important in order to predict how family assistance will react to the emergence of private or public schemes of LTC insurance.

In this paper we consider a setting where children can provide informal care to dependent parents, and intergenerational exchanges are based on a care vs. bequest (or gift) exchange. Children differ in their preferences, which determine their cost (disutility) of providing informal care. This heterogeneity reflects the fact that the level of informal care that can be obtained from the family in case of dependence depends on a number of factors such as the presence of children, their proximity, working situations, marital status, and the strength of family bonds.

Our framework is inspired by the strategic bequest approach, ${ }^{2}$ but it differs from the conventional model in a crucial way in that parents do not perfectly know their children's preferences and their cost of providing informal care. Like in the conventional model we assume that parents can commit to a bequest rule specifying a transfer, gift or bequests, conditional on the level of informal care provided. However, because of the asymmetry of information, this does no longer allow them to extract the full surplus generated by the exchange from their children. Even though parents can use nonlinear rules to screen for the children's cost parameter, they will have to leave a positive rent to some of the children.

In the laissez-faire, the help provided by high-cost children is distorted downwards in order to mitigate the rent enjoyed by the low-cost ones. Parents are not insured against the risk of dependence, nor against the risk that their children have a high cost of providing care.

We then introduce social LTC in this setting. It is designed to maximize a weighted sum of parent's and children's utilities. In other words we explicitly account for the wellbeing of caregivers. This further differentiates our paper from most of the literature, which has to a large extent concentrated on parents' welfare. ${ }^{3}$ To concentrate on LTC

\footnotetext{
${ }^{2}$ See for instance Kotlikoff and Spivak (1981), Bernheim et al. (1985).

${ }^{3}$ Barigozzi et al. (2017) also account for the welfare of caregivers, but consider a different type of
} 
policies we assume throughout the paper that the government cannot make any direct transfers to the children.

In the first part of the paper we consider a uniform social LTC policy. It provides to all dependent individuals a given LTC transfer, which is financed by a uniform lumpsum tax. We show that this policy affects distortions of informal care and thus the distribution of rents between parents and children. The optimal policy then involves a tradeoff between providing insurance to parents and enhancing the utility of the caregivers. In the absence of informal care, social LTC insurance should fully insure the risk of dependence. With informal care and strategic bequests, the optimal public LTC policy depends on the attitude towards risk of parents. Under DARA (decreasing absolute risk aversion) preferences, public LTC insurance exacerbates the distortion of informal care. Better insurance coverage makes dependent parents less reliant on informal care, so that distorting down informal care is not too costly for them. Consequently, under DARA, the optimal public LTC policy provides less than full insurance in order to mitigate the distortion of informal care and the reduction in (low-cost) children's utility it brings about. The opposite is true under IARA (increasing absolute risk aversion) preferences, in which case the government should provide more than full insurance against the risk of dependence in order to minimize distortions.

In the second part of the paper we consider nonlinear policies, where transfers form parents to children are publicly observable and LTC benefits can be conditioned on bequests (or gifts). The LTC policy can then screen for the children's cost parameter, even when the level of informal care is observable only to parents. The underlying problem presents methodological challenges because we have to deal with a "nested" principal-agent problem. While the policy can screen for the children's cost of providing care, this is done only indirectly via the parents. The latter do not observe their child's cost of providing care either but since they observe informal care, they have superior family exchanges based on cooperative bargaining. 
information.

We show that, while with a uniform policy the results crucially depend on parents' risk aversion, this is no longer true when the policy is restricted by informational considerations only and can be nonuniform. In that case the available policy instruments are sufficiently powerful to ensure that parents are always fully insured, even against the risk of having high-cost children. And since they are fully insured, risk aversion no longer matters. Even more strikingly, the tradeoff between the provision of insurance to parents and the concern for the welfare of the caregivers which drives the results for a uniform policy is not longer relevant when nonuniform policies are considered.

One surprising result regarding the impact of social LTC insurance on informal care emerges in both cases. In most of the literature on LTC, crowding out of informal care by public care is considered a serious problem. It makes the provision of social LTC insurance more expensive and possibly even ineffective, when there is full crowding out. If one accounts for the wellbeing of caregivers the impact of crowding out is more complex. One might conjecture that is "bad" for parents, but "good" for children, since it reduces the cost implied by care provision. However, this conjecture does not stand under closer scrutiny, and is proven wrong or misleading by our results. This is because in our setting children are paid for their care through gifts or bequests. Under full information, when care decreases, their compensation is reduced to keep their utility constant. Crowding out is then irrelevant. Under asymmetric information, on the other hand, crowding out will reduce the rents of some of the children and thus effectively decrease their utility, while it increases parents' welfare. Consequently, the concern for the wellbeing of caregivers does not imply that LTC policy should aim at reducing informal care.

The paper is organized as follows. We present the model in Section 2. We study the uniform and the nonlinear LTC policies, in Section 3 and 4, respectively. In Section 5 we provide some concluding remarks. 


\section{Model}

Consider a generation of identical parents. When old they face the risk of being dependent with probability $\pi$, while they are independent and healthy with probability $(1-\pi)$. When young they each have one child, they earn an exogenous labor income of $w \bar{T}$ of which they save $s$. They have preferences over consumption when young, $c \geq 0$, consumption when old and healthy, $d \geq 0$, and consumption, including LTC services, when old and dependent, $m \geq 0$. Their preferences are quasilinear in consumption when young. Risk aversion is introduced through the concavity of second period state dependent utilities. The parents' expected utility is given by

$$
E U=w \bar{T}-s+(1-\pi) U(s)+\pi E[H(m)]
$$

with $m=s+a-\tau(a)$, where $a \geq 0$ is informal care provided by children, while $\tau(a)$ is a transfer (bequest or gift) from parents to children. One can think of $m$ as total care, that is informal care $a$ plus formal care $s-\tau(a)$, the price of which is normalized to one. We assume that the transfer can be conditioned on informal care and assume that parents can commit to this bequest rule. This is in line with the strategic bequest literature. However, our model differs from the traditional literature on exchange based intergenerational transfers in that we assume that parents may not perfectly observe their child's preferences and in particular their cost of providing care. The children's heterogeneity in cost is represented by a parameter $\beta$ which is not publicly observable, including to parents. Assume that $\beta$ is distributed over $\{\underline{\beta}, \bar{\beta}\}$. In other words, $\beta$ can only take two values. The low one $\beta$ occurs with probability $\lambda \in] 0,1[$, while the high level $\bar{\beta}$ occurs with probability $(1-\lambda)$.

Children's cost of providing care $a$ to their parents is given by $v(a, \beta)$, where $0 \leq$ $a \leq a^{\max }$, with $v_{a}>0, v_{\beta}<0, v_{a a}>0, v_{a \beta}<0$, where subscripts denote partial derivatives. ${ }^{4}$ The cost is increasing and convex in the level of informal care. It decreases

\footnotetext{
${ }^{4}$ The assumption that there is an upper bound on informal care is quite natural. Furthermore it is
} 
in $\beta$, which amounts to saying that $\bar{\beta}$ is the "good type" for whom providing care is less costly. Furthermore $v_{a \beta}<0$ implies that the marginal cost of informal care also decreases with $\beta$.

The children's utility from helping their parents in case of dependence is

$$
U_{k}=c_{k}-v(a, \beta) \geq 0
$$

where children's consumption $c_{k}=\tau(a)$, that is the transfer from their parents.

Children choose $a$ to maximize $U_{k}$

$$
\tau^{\prime}(a)=v_{a}(a, \beta)
$$

and the solution to this problem is denoted $a(\beta)$. Observe that $a$ also depends on $\tau(\cdot)$, exactly as labor supply depends on the tax function in a Mirrleesian-type optimal income tax problem.

Anticipating their children's behavior but not observing $\beta$, parents choose $s$ and $\tau(a)$ to maximize their expected utility given by

$$
E U=w \bar{T}-s+(1-\pi) U(s)+\pi E_{\beta}[H(s+a(\beta)-\tau(a(\beta))] .
$$

To solve this problem we consider the equivalent mechanism design problem where parents parents choose $a(\beta)$ and $\tau(\beta)$ to maximize

$$
E U=w \bar{T}-s+(1-\pi) U(s)+\pi E_{\beta}[H(s+a(\beta)-\tau(\beta)]
$$

subject to the relevant participation constraints, as well as the incentive constraints. These constraints which come about when the parents do not observe $\beta$ will be stated and explained below.

Within this framework, we study the public provision of LTC benefits financed by a lump-sum tax on parents' first-period consumption. In addition, intergenerational convenient for technical reasons. 
transfers $\tau$ may be taxed or subsidized. The policy is determined to maximize social welfare which is given by a weighted sum of parents' and children's expected utilities. Parents' weight is $\alpha \in(0,1)$, while children's utility is weighted by $1-\alpha$.

The timing is as follows. The LTC policy is decided upon in stage 1, before parents and children make their decisions. In stage 2, parents choose their level of savings $s$ and commit to the bequest rule $\tau(a)$. In stage 3 , the dependence status of the parents is realized and children choose $a$ according to (1).

While we concentrate on the asymmetric information case, we start by considering the full information benchmark, that is the solution parents can achieve when they observe their children's cost of providing care. We also characterize the optimal LTC policy for this benchmark.

\section{Uniform LTC benefit}

In this section, we restrict the policy to a uniform transfer $g$ financed by a lump-sum tax. In other words, we consider universal public provision (or subsidization) of LTC. Such a policy is clearly suboptimal if the government can condition transfers on bequests. ${ }^{5}$ However, it is relevant in practice, since the government may not be able to observe bequests. Furthermore, even if the government was able to observe bequests, it may be politically infeasible to condition $g$ on $\tau$. In this section, we first characterize the optimal policy when parents can observe their children's type. We then turn to the asymmetric information case.

\subsection{Full information benchmark}

Parent's choose $s$ and commit to a bequest rule ex ante, that is before the state of health and $\beta$ are realized and observed. Since $\beta$ takes only two values, it is convenient

\footnotetext{
${ }^{5}$ In Section 4 we will consider the case where $g$ can be conditioned on bequests, so that the government can screen for different levels of $\beta$.
} 
to introduce the following notation

$$
\begin{array}{cll}
\tau(\bar{\beta})=\bar{\tau} & ; & \tau(\underline{\beta})=\underline{\tau}, \\
a(\bar{\beta})=\bar{a} & ; & a(\underline{\beta})=\underline{a}, \\
m(\bar{\beta})=\bar{m} & ; & \tau(\underline{\beta})=\underline{m} .
\end{array}
$$

Using this notation the parent's problem can be written as

$$
\begin{array}{rl}
\max _{\bar{a}, \underline{a}, \bar{\tau}, \underline{\tau}, s} & w \bar{T}-\pi g-s+(1-\pi) U(s)+\pi[\lambda H(s+\underline{a}-\underline{\tau}+g)+(1-\lambda) H(s+\bar{a}-\bar{\tau}+g)] \\
\text { s.t. } & \underline{\tau}-v(\underline{a}, \underline{\beta}) \geq 0 \\
& \bar{\tau}-v(\bar{a}, \bar{\beta}) \geq 0 .
\end{array}
$$

where $\pi g$ is the lump-sum tax levied to finance the expected cost of social LTC transfers. Conditions (2) and (3) represent the children's participation constraint. While children take the bequest rule $\tau(a)$ as given, they have the option not to exchange with their parents: in this case there will be no care and no transfer and chilrden's utility is an exogenously given constant which without loss of generality is normalized to zero.

Under full information, the parent can extract all the surplus, and both participation constraints are binding. ${ }^{6}$ Then, substituting for $\underline{\tau}$ and $\bar{\tau}$ from (2) and (3) the parent's problem can be rewritten as

$$
\begin{aligned}
\max _{\bar{a}, \underline{a}, s} \quad P^{f}= & w \bar{T}-\pi g-s+(1-\pi) U(s)+\pi[\lambda H(s+\underline{a}-v(\underline{a}, \underline{\beta})+g) \\
& +(1-\lambda) H(s+\bar{a}-v(\bar{a}, \bar{\beta})+g)] .
\end{aligned}
$$

The first-order conditions (FOC) with respect to the remaining choice variables are

\footnotetext{
${ }^{6}$ When a participation constraint is not binding parents can increase the corresponding $a$ and/or decrease $\tau$, thereby increasing their expected utility.
} 
given by

$$
\begin{aligned}
\frac{\partial P^{f}}{\partial \bar{a}} & =(1-\lambda) H^{\prime}(\bar{m})\left[1-v_{a}(\bar{a}, \bar{\beta})\right]=0, \\
\frac{\partial P^{f}}{\partial \underline{a}} & =\lambda H^{\prime}(\underline{m})\left[1-v_{a}(\underline{a}, \underline{\beta})\right]=0, \\
\frac{\partial P^{f}}{\partial s} & =-1+(1-\pi) U^{\prime}(s)+\pi\left[\lambda H^{\prime}(\underline{m})+(1-\lambda) H^{\prime}(\bar{m})\right]=0 .
\end{aligned}
$$

The first two conditions imply

$$
1=v_{a}(\bar{a}, \bar{\beta})=v_{a}(\underline{a}, \underline{\beta}),
$$

which is quite intuitive. Under full information parents have to compensate children exactly for their utility cost of informal care. Consequently, they equalize marginal costs to marginal benefits, which are equal to one. Not surprisingly, this implies $\bar{a}>\underline{a}$ and $\bar{m}>\underline{m}$ : low-cost children provide more informal care and their parents enjoy a larger amount of total care, $m$, in case of dependence. To decentralize this solution parents must then choose $\tau$ so that $\tau^{\prime}(\bar{a})=\tau^{\prime}(\underline{a})=1$. Observe that neither $\bar{a}$ nor $\underline{a}$ depend on $g$. Consequently, a uniform $g$ can never achieve full insurance for the risk associated with the uncertainty of $\beta$; parents with low-cost children are always better off.

We now turn to the government's problem, which is given by

$$
\begin{aligned}
\max _{g} \quad G^{f}= & w \bar{T}-\pi g-s+(1-\pi) U(s)+\pi[\lambda H(s+\underline{a}-v(\underline{a}, \underline{\beta})+g) \\
& +(1-\lambda) H(s+\bar{a}-v(\bar{a}, \bar{\beta})+g)]
\end{aligned}
$$

where $s, \underline{a}$ and $\bar{a}$ are the solutions to the parents' problem for any given $g$. Since the parents have full information, the children's utility will be zero no matter what. Consequently, the relative weight of children in social welfare is of no relevance and we can just as well neglect this term in the welfare function.

Using the envelope theorem (for the induced effect on $s$ ) and recalling that the levels 
of $a$ do not depend on $g$ we have ${ }^{7}$

$$
\frac{\partial G^{f}}{\partial g}=-\pi+\pi\left[\lambda H^{\prime}(\underline{m})+(1-\lambda) H^{\prime}(\bar{m})\right]=0 .
$$

Not surprisingly, this condition equalizes marginal costs and benefits of $g$. Combining this condition with the parent's FOC with respect to $s$ in (4) yields

$$
U^{\prime}(s)=\left[\lambda H^{\prime}(\underline{m})+(1-\lambda) H^{\prime}(\bar{m})\right]=E_{\beta}\left[H^{\prime}(m)\right]=1 .
$$

This condition states that the three possible uses of (first-period) income, direct consumption $c$, deferred consumption $s$, and LTC insurance, $g$ must have the same marginal expected utility.

\subsection{Asymmetric information}

Except for the policy design, the previous section has presented a rather standard strategic bequest model. Parents have all the bargaining power and have full information about their children's cost of providing care. We now turn to the more interesting case where parents do not know their children's type. We then have to add two incentive constraints to the parents' problem. The objective function does not change and the participation constraints continue of course to apply. The parents' problem can then be stated as follows

$$
\begin{aligned}
& \max _{\bar{a}, \underline{a}, \bar{\tau}, \underline{\tau}, s} \quad P^{a s}=w \bar{T}-\pi g-s+(1-\pi) U(s)+\pi[\lambda H(s+\underline{a}-\underline{\tau}+g) \\
& +(1-\lambda) H(s+\bar{a}-\bar{\tau}+g)] \\
& \text { s.t. } \quad \underline{\tau}-v(\underline{a}, \underline{\beta}) \geq 0, \\
& \bar{\tau}-v(\bar{a}, \bar{\beta}) \geq 0, \\
& \bar{\tau}-v(\bar{a}, \bar{\beta}) \geq \underline{\tau}-v(\underline{a}, \bar{\beta}), \\
& \underline{\tau}-v(\underline{a}, \underline{\beta}) \geq \bar{\tau}-v(\bar{a}, \underline{\beta}) .
\end{aligned}
$$

\footnotetext{
${ }^{7}$ We assume that the second order condition holds.
} 
This is a rather standard mechanism design problem and one can easily show that we obtain the "usual" pattern of binding incentive and participation constraints. To be precise, the participation constraint of high-cost children is binding so that

$$
\underline{\tau}=v(\underline{a}, \underline{\beta}) .
$$

Furthermore, the incentive constraint of $\bar{\beta}$, the low-cost type, is binding. Using (9) this condition can be written as

$$
\bar{\tau}=v(\bar{a}, \bar{\beta})+[v(\underline{a}, \underline{\beta})-v(\underline{a}, \bar{\beta})],
$$

where the term in brackets on the RHS represents the rent of type $\bar{\beta}$. In words, because low-cost children can mimic high-cost ones and provide $\underline{a}$ at a lower cost, they have to receive a transfer exceeding the cost of the care they provide. This is particularly interesting from our perspective because it implies that the utility of the caregivers is no longer exogenously given. Some of them now receive a transfers which puts them above their reservation utility level and this transfer may depend on the LTC policy.

Substituting for the transfers from (9) and (10) into the objective function, the parents' problem can then be rewritten as

$$
\begin{aligned}
\max _{\bar{a}, \underline{a}, s} \quad P^{a s}= & w \bar{T}-\pi g-s+(1-\pi) U(s)+\pi[\lambda H(s+\underline{a}-v(\underline{a}, \underline{\beta})+g) \\
& +(1-\lambda) H(s+\bar{a}-v(\bar{a}, \bar{\beta})-v(\underline{a}, \underline{\beta})+v(\underline{a}, \bar{\beta})+g)] .
\end{aligned}
$$

The first-order conditions are given by

$$
\begin{aligned}
& \frac{\partial P^{a s}}{\partial \bar{a}}=(1-\lambda) H^{\prime}(\bar{m})\left[1-v_{a}(\bar{a}, \bar{\beta})\right]=0, \\
& \frac{\partial P^{a s}}{\partial \underline{a}}=\pi\left\{\lambda H^{\prime}(\underline{m})\left[1-v_{a}(\underline{a}, \underline{\beta})\right]-(1-\lambda) H^{\prime}(\bar{m})\left[v_{a}(\underline{a}, \underline{\beta})-v_{a}(\underline{a}, \bar{\beta})\right]\right\}=0, \\
& \frac{\partial P^{a s}}{\partial s}=-1+(1-\pi) U^{\prime}(s)+\pi\left[\lambda H^{\prime}(\underline{m})+(1-\lambda) H^{\prime}(\bar{m})\right]=0 .
\end{aligned}
$$

From equation (12) we obtain $1=v_{a}(\bar{a}, \bar{\beta})$, which is the full information condition for $\bar{\beta}$, the low-cost type; see equation (5). This is the traditional no distortion at the top 
result that, given the quasi-linearity of the utility function, does not only apply to the rule, but also to the actual level of care $\bar{a}$ which is the same as in the full information solution, and continues to be independent of $g$.

Turning to $\underline{a}$, rearranging (13) yields

$$
\left[1-v_{a}(\underline{a}, \underline{\beta})\right]=\frac{(1-\lambda) H^{\prime}(\bar{m})}{\lambda H^{\prime}(\underline{m})}\left[v_{a}(\underline{a}, \underline{\beta})-v_{a}(\underline{a}, \bar{\beta})\right]>0,
$$

where we have used $v_{a \beta}<0$, which implies $\Delta v_{a}=v_{a}(\underline{a}, \underline{\beta})-v_{a}(\underline{a}, \bar{\beta})>0$. Consequently we have $v_{a}(\underline{a}, \underline{\beta})<1$ implying a downward distortion for $\underline{a}$. Intuitively, $\Delta v_{a}>0$ accounts for the fact that the rent of the low-cost type increases with $\underline{a}$. The downward distortion allows parents to mitigate this rent. Equation (15) also implies that $\underline{a}$ depends on $g$, as well as the bequests left to both types, according to (9) and (10). For $g=0$, the solution to the parents' problem yields the laissez-faire allocation.

We now turn to the government's problem. Since the parents no longer have full information, the low-cost children now have a positive utility level. Moreover, their utility is affected by the LTC policy via its impact on the parents' optimization problem. Consequently, the relative weight of children in social welfare is now relevant. When this weight is strictly positive, the LTC policy strikes a balance between providing insurance coverage to parents and the concern for the wellbeing of the caregivers.

The government's problem is given by

$$
\begin{aligned}
\max _{g} \quad G^{a s}= & \alpha\{w \bar{T}-\pi g-s+(1-\pi) U(s)+\pi[\lambda H(s+\underline{a}-v(\underline{a}, \underline{\beta})+g) \\
& +(1-\lambda) H(s+\bar{a}-v(\bar{a}, \bar{\beta})-[v(\underline{a}, \underline{\beta})-v(\underline{a}, \bar{\beta})]+g)]\} \\
& +(1-\alpha) \pi(1-\lambda)[v(\underline{a}, \underline{\beta})-v(\underline{a}, \bar{\beta})],
\end{aligned}
$$

where $\bar{a}, \underline{a}$ and $s$ are determined by the solution to the parents' problem. Observe that parents' utility is expressed as the solution to the reformulated problem (11) which is an unconstrained optimization where the relevant IC and participation constraints have been substituted into the objective function. 
Using the envelope theorem according to which we can neglect the derivatives of parents' utility with respect to $\underline{a}, \bar{a}$, and $s$, the FOC is given by ${ }^{8}$

$$
\begin{aligned}
\frac{\partial G^{a s}}{\partial g}=\alpha & \left.\alpha-\pi+\pi\left[\lambda H^{\prime}(\underline{m})+(1-\lambda) H^{\prime}(\bar{m})\right]\right\} \\
& +\pi(1-\lambda)(1-\alpha)\left[v_{a}(\underline{a}, \underline{\beta})-v_{a}(\underline{a}, \bar{\beta})\right] \frac{\partial \underline{a},}{\partial g}=0 .
\end{aligned}
$$

Recall that the term $\Delta v_{a}=v_{a}(\underline{a}, \underline{\beta})-v_{a}(\underline{a}, \bar{\beta})$ is positive. Observe that the children's utility does not directly depend on $s$ and that $\bar{a}$ does not depend on $g$, nor does the high-costs children's utility, which is always equal to zero, the exogenous reservation utility. Consequently, the only behavioral response to the LTC policy that is relevant in (17) is $\partial \underline{a}, / \partial g$ which measures how $g$ affects the level of care provided by the high-cost children. The sign of this expression will in turn determine how the level of care affects the caregivers' utility.

Consider first the case where the caregivers' utility is not included in social welfare, that is $\alpha=1$. Using the parent's FOC, expression (17) can be written as

$$
U^{\prime}(s)=\left[\lambda H^{\prime}(\underline{m})+(1-\lambda) H^{\prime}(\bar{m})\right]=1 .
$$

This is the same rule as the one under full information that was given by (7). In both cases we have $U^{\prime}(s)=1$ so that the level of $s$ is also the same. However, the levels of $m$ will differ from the full information solution, which in turn implies that the level of $g$ will also in general be different, even though the rule is the same.

We use superscripts $f$ and as to refer to the solutions to the full information and asymmetric information problems, specified by (6) and (16) respectively. Suppose that $g^{a s}=g^{f}$. Then, $\lambda H^{\prime}\left(\underline{m}^{a s}\right)+(1-\lambda) H^{\prime}\left(\bar{m}^{a s}\right)>1$, because $\underline{m}^{a s}<\underline{m}^{f}$ (as $\underline{a}$ is distorted downward) and $\bar{m}^{a s}<\bar{m}^{f}$ (as low-cost children receive a positive rent); consequently we must have $g^{a s}>g^{f}$ : the optimal level of LTC benefits is larger under asymmetric information than under full information. Intuitively, $g$ is higher to compensate in part for the downward distortion in $a$ that parents create to mitigate children's rents.

\footnotetext{
${ }^{8}$ We assume that the second order condition holds.
} 
Let us now turn to the case where $\alpha<1$, which includes the utilitarian case where $\alpha=1 / 2$. In this case $g$ is no longer solely determined to provide insurance to parents. The optimal LTC policy also accounts for the impact of $g$ on informal care and thus on children's utility (rents). Roughly speaking, when $\partial \underline{a} / \partial g>0$ one can expect that the effect described for $\alpha=1$ is reinforced by the effect of $g$ on children's rents. Since rents increase in $\underline{a}$, increasing $g$ increases rents. In this case we have $g^{a s}>g^{f}$. Conversely, when $\partial \underline{a} / \partial g<0$, the two effects go in opposite directions. Either way this discussion shows that as soon as $\alpha<1$ the results will crucially depend on the sign of $\partial \underline{a} / \partial g$. The study of this sign requires a closer look at the comparative statics of the parents' problem under asymmetric information. The following lemma is established in Appendix A.1.

Lemma 1 When the parents' utility in case of dependence $H(m)$ exhibits DARA we have $\partial \underline{a} / \partial g<0$; when $H(m)$ exhibits IARA we have $\partial \underline{a} / \partial g>0$.

This lemma shows that the effect of $g$ (via the parents' problem) on the level of care provided by the high-cost children depends on the parents' attitude towards risk. Intuitively our results can be understood as follows. With DARA, as $g$ increases, parents become less risk averse. Then, reducing $m$ in the bad state of nature becomes less costly for them, and distorting $\underline{a}$ downwards becomes more attractive. The case with IARA is exactly symmetrical. Note that empirically DARA appears to receive more support (Friend and Blume, 1975).

Using equation (17) and Lemma 1 we can study the effect of $\alpha$ on $g^{a s}(\alpha)$. For instance, we can compare the utilitarian level $g^{a s}(1 / 2)$ with $g^{a s}(1)$, the level achieved when children are not accounted for in the social welfare function. With DARA we know from Lemma 1 that $\underline{a}$ decreases as $g$ increases, which in turn implies that the utility of the low-cost child decreases; recall that $\Delta v_{a}>0$. Consequently equation (17) evaluated at $g^{a s}(1)$ is negative so that $g^{a s}(1 / 2)<g^{a s}(1)$. Under IARA these effects are reversed and we obtain $g^{a s}(1 / 2)>g^{a s}(1)$. This result also goes through for intermediate levels 
of $\alpha$. Totally differentiating (17) and rearranging yields

$$
\frac{\partial g}{\partial \alpha}=\frac{\frac{(1-\lambda)}{\alpha}\left[v_{a}(\underline{a}, \underline{\beta})-v_{a}(\underline{a}, \bar{\beta})\right] \frac{\partial \underline{a}}{\partial g}}{S O C},
$$

which has the opposite sign of $\partial \underline{a} / \partial g$. Accordingly, under DARA $g$ increases in $\alpha$, while it decreases as $\alpha$ increases under IARA.

Using the FOC of the parents with respect to $s$, (14), equation (17) implies that, whenever $\alpha<1$

$$
U^{\prime}(s)<1<E_{\beta}\left(H^{\prime}(m)\right) \quad \text { if } \quad \partial \underline{a} / \partial g<0,
$$

and

$$
U^{\prime}(s)>1>E_{\beta}\left(H^{\prime}(m)\right) \quad \text { if } \quad \partial \underline{a} / \partial g>0 .
$$

Intuitively, the utility of dependent parents is distorted down with respect to the full information case under DARA. In this case, providing full insurance against the risk of dependence would push parents to cut the utility of low-cost children (by distorting down $\underline{a}$ ). From the perspective of social welfare, there is a then a tradeoff between insurance and children's utility, leading to less than full insurance. Accordingly, parents have an incentive to save more and this increases their consumption if healthy. Under IARA these effects are reversed.

The results obtained in this section are summarized in the following proposition.

Proposition 1 Consider the case where the children's cost of providing care is not observable and where public policy is restricted to a uniform LTC benefit $g$ financed by a lump-sum tax. Informal care is observable only by parents. The optimal LTC policy is such that:

(i) The risk of having children with a high cost of providing care is not fully insured;

(ii) If children's utility has no weight in social welfare, parents are fully insured against dependence. This is achieved through a uniform benefit that is larger than in the full information case. 
(iii) If the weight of children in social welfare is strictly positive, and the parent's utility in case of dependence $H(m)$ exhibits DARA, parents are less than fully insured against dependence. The uniform benefit decreases with the weight of children in social welfare.

(iv) If the weight of children in social welfare is strictly positive, and $H(m)$ exhibits IARA, parents are more than fully insured against dependence. The uniform benefit is higher than under full information and it increases with the weight of children in social welfare.

\section{Nonlinear policies}

We now consider nonlinear policies under which the level of LTC transfers $g$ can be conditioned on $\tau$, which is publicly observable. The LTC policy can then screen for the children's cost parameter $\beta$. We continue to assume that $a$ is observable only to parents. The underlying problem presents methodological challenges because we have to deal with a "nested" principal-agent problem. ${ }^{9}$ While the policy can screen for $\beta$, this is done only indirectly via the parents. The latter do not observe their child's $\beta$ either but since they observe informal care, they have superior information.

We proceed exactly like in the previous section. We start with the full information solution and then concentrate on the case where neither the parents nor the government can observe the children's type $\beta .^{10}$ Parents observe $a$ but the government does not. The policy we study consists of a menu of LTC benefits and bequests pairs. Under asymmetric information parents self-select into a benefit-bequest pair. We will also continue to assume that the government cannot make any direct transfer to children.

Observe that, while we study a mechanism design problem, the policy can be imple-

\footnotetext{
${ }^{9}$ The problem considered by Guesnerie and Laffont (1978) has a similar structure. They analyze nonlinear taxation of a monopolist that in turn uses nonlinear pricing.

${ }^{10}$ The "intermediate" case where only parents have full information is also of some interest. However, since the insight it provides is not directly related to our main results, we restrict ourselves to presenting it in an Appendix.
} 
mented by a suitably designed mix of LTC benefits and taxes. The mix depends on the precise timing and more specifically on whether $\tau$ is interpreted as a gift or a bequest. So far we have been agnostic about this because it was of no relevance. When $\tau$ is a gift and thus precedes the public transfer $g$, the solution can be implemented simply by a function $g(\tau)$ conditioning LTC benefits on $\tau$. When $\tau$ is a bequest, which by definition occurs after $g$ is consumed, we can condition LTC on a reported (planned) $\tau$, but then we have to make sure that parents stick to one of the pairs $(\underline{\tau}, \underline{g})$ or $(\bar{\tau}, \bar{g})$. In particular we have to prevent parents from picking a pair, but then leaving a larger bequest (in order to "buy" more care). This can be done by a nonlinear tax on bequest which is prohibitively large when $\tau$ deviates from the one associated with the level of public LTC consumed. In practice this means that "excess" public transfers can be recovered from an individual's bequest.

\subsection{Full information solution}

In this section, we assume that both parents and the government have full information concerning the children's types. Transfer $\tau$ are also publicly observable. However, $a$ is observed by parents only. The government sets $\bar{g}, \underline{g}, \bar{\tau}, \underline{\tau}$, anticipating the choices of the parents. Parents choose $s$ ex ante to maximize their expected utility, and set a such that $\tau-v(a, \beta)=0$; we can thus define $a^{f}(\tau, \beta)$ as the solution to this equation. We have

$$
\begin{aligned}
& \frac{\partial a^{f}}{\partial \tau}=\frac{1}{v_{a}} \\
& \frac{\partial a^{f}}{\partial \beta}=-\frac{v_{\beta}}{v_{a}}>0 .
\end{aligned}
$$

Parents also . 
The government now maximizes

$$
\begin{aligned}
\max _{\bar{g}, \underline{g}, \bar{\tau}, \underline{\tau}} \quad G^{f f}= & w \bar{T}-\pi(\lambda \underline{g}+(1-\lambda) \bar{g})-s+(1-\pi) U(s) \\
& +\pi\left[\lambda H\left(s+a^{f}(\underline{\tau}, \underline{\beta})-\underline{\tau}+\underline{g}\right)+(1-\lambda) H\left(s+a^{f}(\bar{\tau}, \bar{\beta})-\bar{\tau}+\bar{g}\right)\right] .
\end{aligned}
$$

The FOCs of the government are

$$
\begin{aligned}
& \frac{\partial G^{f f}}{\partial \bar{g}}=-\pi(1-\lambda)+\pi(1-\lambda) H^{\prime}(\bar{m})=0, \\
& \frac{\partial G^{f f}}{\partial \underline{g}}=-\pi \lambda+\pi \lambda H^{\prime}(\underline{m})=0 \\
& \frac{\partial G^{f f}}{\partial \bar{\tau}}=\pi(1-\lambda) H^{\prime}(\bar{m})\left[\frac{\partial a^{f}(\bar{\tau}, \bar{\beta})}{\partial \tau}-1\right]=0, \\
& \frac{\partial G^{f f}}{\partial \underline{\tau}}=\pi \lambda H^{\prime}(\underline{m})\left[\frac{\partial a^{f}(\underline{\tau}, \underline{\beta})}{\partial \tau}-1\right]=0,
\end{aligned}
$$

and parents choose $s$ so that

$$
\frac{\partial G^{f f}}{\partial s}=-1+(1-\pi) U^{\prime}(s)+\pi\left[\lambda H^{\prime}(\underline{m})+(1-\lambda) H^{\prime}(\bar{m})\right]=0 .
$$

Combining these equations yields

$$
H^{\prime}(\bar{m})=H^{\prime}(\underline{m})=U(s)=1,
$$

and

$$
v_{a}(\bar{a}, \bar{\beta})=v_{a}(\underline{a}, \underline{\beta})=1 .
$$

These expressions have a simple interpretation. With full information, a nonuniform LTC policy can provide full insurance not only against the risk of dependence, but also against the risk of having high-cost children. Informal care $a$ is set at the efficient levels for each type of children. We now turn to the case where neither parents nor the government observe the children's types. The "intermediate" case where only parents have full information is also of some interest. It is presented in Appendix A.2, which can be skipped without affecting the readability of the following sections. 


\subsection{Asymmetric information}

When neither the parents nor the government can observe the children's types, the government proposes a menu $((\bar{\tau}, \bar{g}),(\underline{\tau}, \underline{g}))$. The only choice left to parents is to fix the level of $a$ associated with each option. As long as $\bar{\tau}>\underline{\tau}$, parents set these levels of informal care such that the participation constraint of $\beta$ and the incentive constraint of $\bar{\beta}$ are satisfied. Formally, the levels of $a$ are defined by

$$
\underline{\tau}=v\left(\underline{a}^{a s}, \underline{\beta}\right),
$$

and

$$
\bar{\tau}=v\left(\bar{a}^{a s}, \bar{\beta}\right)+v\left(\underline{a}^{a s}, \underline{\beta}\right)-v\left(\underline{a}^{a s}, \bar{\beta}\right) .
$$

The optimal (nonuniform) LTC policy is then determined by solving the following problem ${ }^{11}$

$$
\begin{aligned}
\max _{\bar{g}, \underline{,}, \bar{\tau}, \underline{\tau}} \quad G^{a a}= & \alpha\{w \bar{T}-\pi(\lambda \underline{g}+(1-\lambda) \bar{g})-s+(1-\pi) U(s) \\
& \left.+\pi\left[\lambda H\left(s+\underline{a}^{a s}-\underline{\tau}+\underline{g}\right)+(1-\lambda) H\left(s+\bar{a}^{a s}-\bar{\tau}+\bar{g}\right)\right]\right\} \\
& +(1-\alpha) \pi(1-\lambda)\left[v\left(\underline{a}^{a s}, \underline{\beta}\right)-v\left(\underline{a}^{a s}, \bar{\beta}\right)\right] \\
\text { s.t. } \quad \underline{\tau} & =v\left(\underline{a}^{a s}, \underline{\beta}\right), \\
& \bar{\tau} \\
& =v\left(\bar{a}^{a s}, \bar{\beta}\right)+v\left(\underline{a}^{a s}, \underline{\beta}\right)-v\left(\underline{a}^{a s}, \bar{\beta}\right), \\
& \bar{\tau} \geq \underline{\tau} .
\end{aligned}
$$

To solve this problem, we will first ignore constraint (26). We will then verify ex post if the solution to the unconstrained problem fulfils this constraint. If this is the case,

${ }^{11}$ We will ignore for the moment the IC constraints of the parents, which are given by

$$
\begin{aligned}
& \lambda H\left(s+\underline{a}^{a s}-\underline{\tau}+\underline{g}\right)+(1-\lambda) H\left(s+\bar{a}^{a s}-\bar{\tau}+\bar{g}\right) \geq H\left(s+\underline{a}^{a s}-\underline{\tau}+\underline{g}\right) \\
& \lambda H\left(s+\underline{a}^{a s}-\underline{\tau}+\underline{g}\right)+(1-\lambda) H\left(s+\bar{a}^{a s}-\bar{\tau}+\bar{g}\right) \geq H\left(s+a^{a s}(\bar{\tau}, \underline{\beta})-\bar{\tau}+\bar{g}\right),
\end{aligned}
$$

where $\bar{\tau}=v\left(a^{a s}(\bar{\tau}, \underline{\beta}), \underline{\beta}\right)$. In words, since parents have private information on the level of informal care, the social planner has to ensure that they will not prefer a contract over the other, and propose a pooling contract to the children. In Footnote 12, we show that these constraints are indeed not binding. 
we can indeed ignore the constraint. If not a pooling equilibrium emerges. Substituting (24) and (25) in the objective function, the problem can be rewritten as

$$
\begin{aligned}
\max _{\bar{g}, \underline{a}, \bar{a}, \underline{a}} \quad G^{a a}= & \alpha\{w \bar{T}-\pi(\lambda \underline{g}+(1-\lambda) \bar{g})-s+(1-\pi) U(s) \\
& +\pi \lambda H(s+\underline{a}-v(\underline{a}, \underline{\beta})+\underline{g}) \\
& +\pi(1-\lambda) H(s+\bar{a}-v(\bar{a}, \bar{\beta})-v(\underline{a}, \underline{\beta})+v(\underline{a}, \bar{\beta})+\bar{g})\} \\
& +(1-\alpha) \pi(1-\lambda)[v(\underline{a}, \underline{\beta})-v(\underline{a}, \bar{\beta})] .
\end{aligned}
$$

The FOCs are given by

$$
\begin{aligned}
\frac{\partial G^{a a}}{\partial \bar{g}} & =-\pi(1-\lambda)+\pi(1-\lambda) H^{\prime}(\bar{m})=0 \\
\frac{\partial G^{a a}}{\partial \underline{g}} & =-\pi \lambda+\pi \lambda H^{\prime}(\underline{m})=0 \\
\frac{\partial G^{a a}}{\partial \bar{a}} & =\pi(1-\lambda) H^{\prime}(\bar{m})\left[1-v_{a}(\bar{a}, \bar{\beta})\right]=0 \\
\frac{\partial G^{a a}}{\partial \underline{a}} & =\pi \alpha \lambda H^{\prime}(\underline{m})\left[1-v_{a}(\underline{a}, \underline{\beta})\right]-\pi(1-\lambda)\left[\alpha H^{\prime}(\bar{m})-(1-\alpha)\right]\left[v_{a}(\underline{a}, \underline{\beta})-v_{a}(\underline{a}, \bar{\beta})\right]=0
\end{aligned}
$$

Conditions (27) and (28), combined with the parents' FOC with respect to savings imply that

$$
U^{\prime}(s)=H^{\prime}(\underline{m})=H^{\prime}(\bar{m})=1
$$

Then, we have $\underline{m}=\bar{m}$ implying that, under asymmetric information, the optimal nonuniform LTC insurance scheme provides full insurance not only against the risk of dependence, but also against the uncertainty associated with informal care. This is in stark contrast with the results obtained with a uniform policy where full insurance could not be achieved.

Informal care is set at its first best level for low-cost children, as it is shown in (29). Conversely, the optimal level of informal care provided by high-cost children is distorted. Combining (29) with (28) and (30) shows that an interior solution for $\underline{a}$ is determined 
by

$$
\lambda \alpha\left[1-v_{a}(\underline{a}, \underline{\beta})\right]-(1-\lambda)(2 \alpha-1)\left[v_{a}(\underline{a}, \underline{\beta})-v_{a}(\underline{a}, \bar{\beta})\right]=0 .
$$

Since $v_{a}$ decreases in $\beta$, the direction of the distortion depends on the sign of $(2 \alpha-1)$. If children have a lower weight than parents in the social welfare function $(\alpha>1 / 2)$, then $\underline{a}$ is distorted downward. If parents and children have the same weight, $(\alpha=1 / 2)$, there will be no distortion of informal care. Intuitively, the rent is purely a transfer in this case, since the social marginal utility of consumption is the same for children and parents. Finally, if children have a higher weight than parents, there will be an upward distortion of informal care.

The optimal LTC transfer is higher the lower the level of bequests (and the lower the level of informal care). To see this, consider the allocation characterized by (27)-(30). Since individuals are fully insured, $\underline{m}=\bar{m}$, which implies

$$
g(\bar{\tau}) \leq g(\underline{\tau}) \Longleftrightarrow \bar{a}-\bar{\tau} \geq \underline{a}-\underline{\tau} .
$$

Using the IC and PC of the children, this can be rewritten as

$$
g(\bar{\tau}) \leq g(\underline{\tau}) \Longleftrightarrow \bar{a}-v(\bar{a}, \bar{\beta}) \geq \underline{a}-v(\underline{a}, \bar{\beta}),
$$

which is always true since $\bar{a}=\arg \max _{x} x-v(x, \bar{\beta})$. Then, the optimal separating policy $g(\tau)$ implies higher transfers to the parents of high-cost children, in order to compensate them for the lower level of informal care they receive. ${ }^{12}$

The allocation characterized above is a solution to the government's problem only if it satisfies (26). Denote $\underline{a}^{*}$ and $\bar{a}^{*}$ the solutions to (31) and (29). Differentiating (31) shows that $\underline{a}^{*}$ always decreases in $\alpha$ (and thus increases in children's weight $1-\alpha$ ), irrespective of the degree of risk aversion of the parents. ${ }^{13}$ If children have no weight in

\footnotetext{
${ }^{12}$ This solution implies $\underline{m}=\bar{m}$ so that the parents' IC constraints (22) and (23) stated in Footnote 11 are satisfied.

${ }^{13}$ Differentiating (31), one obtains that the sign of $\partial \underline{a}^{*} / \partial \alpha$ is equal to the sign of

$$
\lambda\left[1-v_{a}(\underline{a}, \underline{\beta})\right]-2(1-\lambda)\left[v_{a}(\underline{a}, \underline{\beta})-v_{a}(\underline{a}, \bar{\beta})\right],
$$

which is always negative under (31) since $(2 \alpha-1) / \alpha \leq 1$ for all $\alpha \leq 1$.
} 
the social welfare function $(\alpha=1)$, then $\underline{a}$ is distorted downwards, so that $\underline{a}^{*}<\bar{a}^{*}$. If $\alpha=0$, then the LHS of (31) is always positive. In this case there is a corner solution with $\underline{a}^{*}=a^{\max } \geq \bar{a}^{*}$, where $a^{\max }$ is the maximum level of informal care that can be provided by children. Then, there exist a threshold $\widehat{\alpha}=(1-\lambda) /(2-\lambda)<1 / 2$ such that the optimal policy implies $\underline{a}=\underline{a}^{*} \leq \bar{a}^{*}$ if and only if $\alpha \geq \widehat{\alpha} \cdot{ }^{14}$ Using (24) and (25) it follows that this solution satisfies constraint (26).

When $\alpha<\widehat{\alpha}$ we have $\underline{a}^{*}>\bar{a}^{*}$, which violates constraint (26), since

$$
\underline{\tau}=v\left(\underline{a}^{*}, \underline{\beta}\right)>v\left(\bar{a}^{*}, \bar{\beta}\right)+v\left(\underline{a}^{*}, \underline{\beta}\right)-v\left(\underline{a}^{*}, \bar{\beta}\right)=\bar{\tau},
$$

whenever $\underline{a}^{*}>\bar{a}^{*}$. In this case, the optimal policy consists in a pooling contract $\left\{\tau^{p}, g^{p}\right\}$. Observe that, since $\widehat{\alpha}<1 / 2$, pooling requires that children receive a larger weight than parents in social welfare.

To complete the analysis let us now determine this pooling equilibrium. Under this contract parents set $a^{p}$ such that $\tau^{p}=v\left(a^{p}, \underline{\beta}\right)$ and the government's problem can be written as

$$
\begin{aligned}
\max _{g, a} \quad G^{a a p}= & \alpha\{w \bar{T}-\pi g-s+(1-\pi) U(s)+\pi H(s+a-v(a, \underline{\beta})+g)\} \\
& +(1-\alpha) \pi(1-\lambda)[v(a, \underline{\beta})-v(a, \bar{\beta})] .
\end{aligned}
$$

The FOCs are given by

$$
\begin{aligned}
& \frac{\partial G^{\text {aap }}}{\partial g}=-\pi+\pi H^{\prime}=0, \\
& \frac{\partial G^{\text {aap }}}{\partial a}=\pi \alpha H^{\prime}\left[1-v_{a}(a, \underline{\beta})\right]+\pi(1-\lambda)(1-\alpha)\left[v_{a}(a, \underline{\beta})-v_{a}(a, \bar{\beta})\right]=0 .
\end{aligned}
$$

Condition (32) yields $H^{\prime}=1$. This, combined with the FOC of the parents, implies $1=U^{\prime}(s)=H^{\prime}(m)$ so that we continue to have full insurance against both the risk of dependence and the risk of having a high-cost child. Furthermore, (33) can be rewritten as

$$
\left[1-v_{a}\left(a^{p}, \underline{\beta}\right)\right]+(1-\lambda) \frac{(1-\alpha)}{\alpha}\left[v_{a}\left(a^{p}, \underline{\beta}\right)-v_{a}\left(a^{p}, \bar{\beta}\right)\right]=0 .
$$

\footnotetext{
${ }^{14}$ The threshold $\widehat{\alpha}$ is the value of $\alpha$ such that (31) is satisfied for $\underline{a}=\bar{a}^{*}$.
} 
Differentiating this expression and making use of the SOC show that $\partial a^{p} / \partial \alpha<0$ so that under the pooling contract informal care continues to increase with the weight of children in the social welfare function.

The main results of this section are summarized in the following proposition.

Proposition 2 Consider the case where children's cost of providing care is not observable and where LTC benefits $g$ can be conditioned on the transfer $\tau$ paid by parents to children in exchange for informal care. Informal care is observable only to parents. The optimal LTC policy may involve a separating or a pooling contract. This policy is such that:

(i) The risk of having high-cost children is fully insured.

(ii) The average level of informal care always increases in the weight of children in the social welfare function, irrespective of the parents' degree of risk aversion.

(iii) A separating contract is optimal if and only if $\alpha \geq(1-\lambda) /(2-\lambda)<1 / 2$. It implies that:

(a) Informal care is set at its first best level for the low-cost children.

(b) The level of informal care provided by high-cost children is distorted and the direction of the distortion depends on children's weight in the welfare function. It has the same sign as $(2 \alpha-1)$ so that a downward (upward) distortion occurs when the weight of the children is lower (higher) than the weight of parents.

Note that, while with a uniform policy the results crucially depend on parents' risk aversion, this is no longer true when the policy is restricted by informational considerations only, and can be nonuniform. In that case the available policy instruments are sufficiently powerful to ensure that parents are always fully insured, even against the risk of having high-cost children. And since they are fully insured, risk aversion no 
longer matters. Even more strikingly, the tradeoff between the provision of insurance to parents and the concern for the welfare of the caregivers which drives the results for a uniform policy is no longer relevant under nonuniform policies.

\section{Conclusion}

We study the design of long-term care (LTC) policy when informal care from children to dependent parents is due to a bequest motive. Parents do not observe their children's cost of providing care, but they can commit to a bequests rule specifying a transfer conditional on the level of informal care. The social welfare function is a weighted sum of parents' and children's utility. We show that social LTC insurance affects the exchanges between parents and children and in particular the level of informal care and the distribution of rents.

The optimal uniform public LTC insurance depends on the attitude towards risk of parents. Under DARA (decreasing absolute risk aversion) preferences, public LTC insurance exacerbates the distortion of informal care. Consequently, the optimal public LTC coverage provides less than full insurance. The opposite is true under IARA (increasing absolute risk aversion) preferences. A uniform policy can never insure the risk of having a high-cost child.

A nonuniform policy that conditions LTC benefits on bequests provides full insurance even for the risk of having high-cost children. The level of informal care provided by high-cost children is distorted and the direction of the distortion depends on children's weight in the social welfare function.

Interestingly, in the uniform as well as in the nonuniform case, the higher the weight of children in the social welfare function, the higher the optimal average level of informal care. This apparently counter-intuitive result is due to the exchange motive behind family help. Under this motive, higher reliance on the family for the provision of longterm care implies higher rents for children. In our model, crowding out of family help by 
public care only affects social welfare through its (negative) effect on children's utility. A main lesson that emerges from our analysis is that in an exchange-based setting, social insurance should be designed in order to ensure that dependent elderly have to rely even on high-cost children. This ensures that low-cost children get rewarded for the informal care they provide.

Another major lesson is that, even with ex ante identical individuals, the nonuniform policy performs better and is able to provide full insurance against both underlying risks. In other words, even with identical individuals, social LTC should involve some measure of means testing and/or recover part of the benefits received by the elderly from their estate. This results is interesting because means testing is usually justified by redistribution. We have not considered redistributive motive, but they could only be expected to reinforce this result.

Throughout the paper we have remained agnostic about the exact nature of the transfer, gift or bequest, that "pays" for the care, except that we have pointed out that it affects the timing of the underlying game. From and empirical perspective, however, the gift interpretation appears to be more compelling. The literature has found some evidence that inter vivos transfers are larger for those children who provide informal care (Norton and Van Houtven, 2006). However, these estimates are often problematic because of endogeneity problems and because measurement of both informal care and financial transfers within the family is difficult. More reliable estimates require longitudinal data and a step in that direction is taken by Norton et al. (2014) who use the 1999 and 2003 waves of National Longitudinal Survey of Mature Women and show that children providing informal care are indeed more likely to receive financial transfers from their parents.

Considering bequests as payment for care is more problematic because research has shown that bequests are typically divided equally among children (Menchik, 1980; Tomes, 1981). Even when children provide unequal amounts of informal care, bequests 
tend to be divided equally (Norton and Taylor, 2005). This is true for the US but the argument is even more compelling for most European countries, where equal sharing rules are imposed by law. In any event there are also theoretical arguments that favor inter vivos transfers over bequests as payment for informal care. Gifts are more flexible and, as argued by Norton and Van Houtven (2006), "can be adjusted quickly to the amount of care, are less costly than writing a will, and can be kept secret from other family members and the public".

Our policy recommendations are made under the assumption that the provision of informal care is exchange-based. This hypothesis has received empirical support and appears to apply to certain families. Roughly speaking, “... the idea of exchange makes sense for those extended families where an older person has money and needs help, and a younger person has time and needs money", (Norton et al. 2014). But it is clearly not the only behavioral pattern that is relevant. In reality the different types of intra-family relations, based on altruism, norms or selfish exchanges, with and without commitment are likely to coexist. ${ }^{15}$ The different studies provide partial and intermediate answers which can provide valuable guidance for the design of social LTC policy, as long as interpreted with suitable care and keeping in mind the underlying assumptions.

\footnotetext{
${ }^{15}$ Alternative approaches explored for instance by Cremer and Roeder (2017) Barrigozzi et al. (2017), Canta and Pestieau (2014), Ponthière (2014).
} 


\section{References}

[1] Barigozzi, F., H. Cremer and K. Roeder, (2017), "Caregivers in the family: daughters, sons and social norms," TSE-Working Paper.

[2] Bernheim, B., A. Shleifer and L. Summers, (1985), "The strategic bequest motive", Journal of Political Economy, 93, 1045-1076.

[3] Colombo, F., A. Llena-Nozal, J. Mercier and F. Tjadens, (2011), "Help Wanted? Providing and paying for long-term care", OECD Health Policy Studies, OECD Publishing.

[4] Canta, C. and P. Pestieau, (2014), "Long-Term Care Insurance and Family Norms", BEJEAP, 14, 401-429.

[5] Cremer, H., P. Pestieau and G. Ponthière, (2012), "The economics of long-term care: A survey", Nordic Economic Policy Review, 2, 107-148.

[6] Cremer, H. and K. Roeder, (2017), "Long-term care policy with lazy rotten kids", Journal of Public Economic Theory, forthcoming.

[7] Coe, N. B. and C. H. Van Houtven, (2009), "Caring for Mom and Neglecting Yourself? The Health Effects of Caring for an Elderly Parent." Health Economics, 18, 991-1010.

[8] Friend, I. and M. Blume, (1975), "The Demand for Risky Assets", American Economic Review, 65, 900-922.

[9] Guesnerie, R. and J.-J. Laffont, (1978), "Taxing price makers", Journal of Economic Theory, 19, 423-455.

[10] Kotlikoff, L. and A. Spivak (1981) "The Family as an Incomplete Annuities Market." Journal of Political Economy, 89, 372-91. 
[11] Menchik, P. L., (1980), "Primogeniture, Equal Sharing and the U.S. Distribution of Wealth." Quarterly Journal of Economics, 94, 299-316.

[12] Norton, E., (2000), "Long term care", in A. Cuyler \& J. Newhouse (Eds.): Handbook of Health Economics, Volume 1b, chapter 17.

[13] Norton, E., H. Nicholas and S. Huang, (2014), Informal Care and Inter-vivos Transfers: Results from the National Longitudinal Survey of Mature Women, BEJEAP, $14,377-401$.

[14] Norton, E. C. and D. H. Taylor, Jr, (2005), "Equal Division of Estates and the Bequest Motive." Journal of Aging and Social Policy, 17, 63-82.

[15] Norton, E. C. and C. H. Van Houtven, (2006), "Inter-vivos Transfers and Exchange." Southern Economic Journal 73, 157-72.

[16] Ponthière, G., (2014), "Long-Term Care, Altruism and Socialization", BEJEAP, $14,429-473$.

[17] Tomes, N., (1981) "The Family, Inheritance and the Intergenerational Transmission of Inequality." Journal of Political Economy, 89, 928-58. 


\section{Appendix}

\section{A.1 Proof of Lemma 1}

Since $\bar{a}$ is independent off $g$, we can focus on the FOCs (13) and (14) to study the comparative statics with respect to $g$. Using subscripts to denote partial derivative, define

$$
H=\left[\begin{array}{ll}
P_{a a}^{a s} & P_{s a}^{a s} \\
P_{\underline{a} s}^{a s} & P_{s s}^{a s}
\end{array}\right]
$$

and

$$
D=\left[\begin{array}{c}
-P_{a g}^{a s} \\
-P_{s g}^{a s}
\end{array}\right]
$$

where

$$
\begin{aligned}
& P_{s \underline{a}}^{a s}=P_{\underline{a} s}^{a s}=\pi\left[\lambda H^{\prime \prime}(\underline{m})\left(1-v(\underline{a}, \underline{\beta})-(1-\lambda) H^{\prime \prime}(\bar{m})\left(v_{a}(\underline{a}, \underline{\beta})-v_{a}(\underline{a}, \bar{\beta})\right)\right]=\pi A,\right. \\
& P_{s s}^{a s}=(1-\pi) U^{\prime \prime}(s)+\pi\left[\lambda H^{\prime \prime}(\underline{m})+(1-\lambda) H^{\prime \prime}(\bar{m})\right], \\
& P_{\underline{a} g}^{a s}=\pi\left\{\lambda H^{\prime \prime}(\underline{m})\left[1-v_{a}(\underline{a}, \underline{\beta})\right]-(1-\lambda) H^{\prime \prime}(\bar{m})\left[v_{a}(\underline{a}, \underline{\beta})-v_{a}(\underline{a}, \bar{\beta})\right]\right\}=\pi A, \\
& P_{s g}^{a s}=\pi\left[\lambda H^{\prime \prime}(\underline{m})+(1-\lambda) H^{\prime \prime}(\bar{m})\right],
\end{aligned}
$$

and where

$$
A=\lambda H^{\prime \prime}(\underline{m})\left[1-v_{a}(\underline{a}, \underline{\beta})\right]-(1-\lambda) H^{\prime \prime}(\bar{m})\left[v_{a}(\underline{a}, \underline{\beta})-v_{a}(\underline{a}, \bar{\beta})\right] .
$$

Using Cramer's rule we obtain

$$
\frac{\partial \underline{a}}{\partial g}=\frac{\left|\begin{array}{cc}
-P_{a g}^{a s} & P_{s a}^{a s} \\
-P_{s g}^{a s} & P_{s s}^{a s}
\end{array}\right|}{|H|}
$$

where $|H|>0$ from the SOC.

Substituting from (A.1)-(A.4), evaluating the determinant and simplifying successively yields

$$
\begin{aligned}
\operatorname{sgn}\left(\frac{\partial \underline{a}}{\partial g}\right) & =\operatorname{sgn}\left(\left|\begin{array}{cc}
-A \pi & A \pi \\
\pi\left[\lambda H^{\prime \prime}(\underline{m})+(1-\lambda) H^{\prime \prime}(\bar{m})\right] & (1-\pi) U^{\prime \prime}(s)+\pi\left[\lambda H^{\prime \prime}(\underline{m})+(1-\lambda) H^{\prime \prime}(\bar{m})\right]
\end{array}\right|\right) \\
& =\operatorname{sgn}\left(-\pi A\left[(1-\pi) u^{\prime \prime}(s)\right]\right)=\operatorname{sgn}(A) .
\end{aligned}
$$


To sum up we have to study the sign of $A$ defined by (A.5). Substituting from (15) and rearranging yields

$$
A=(1-\lambda) \Delta v_{A}\left[H^{\prime \prime}(\underline{m}) \frac{H^{\prime}(\bar{m})}{H^{\prime}(\underline{m})}-H^{\prime \prime}(\bar{m})\right] .
$$

Because $\Delta v_{A}>0$, this expression has the same sign as the term in brackets on the RHS.

Consequently we have

$$
\begin{aligned}
A>0 & \Longleftrightarrow \quad \frac{H^{\prime \prime}(\underline{m})}{H^{\prime}(\underline{m})}>\frac{H^{\prime \prime}(\bar{m})}{H^{\prime}(\bar{m})} \\
& \Longleftrightarrow \quad-\frac{H^{\prime \prime}(\underline{m})}{H^{\prime}(\underline{m})}<-\frac{H^{\prime \prime}(\bar{m})}{H^{\prime}(\bar{m})} .
\end{aligned}
$$

Since $\underline{m}<\bar{m}$ this is true under IARA (Increasing Absolute Risk Aversion), while DARA (Decreasing Absolute Risk Aversion) yields $A<0$.

\section{A.2 Only parents have full information}

If the government does not observe $\beta$, but parents do, the problem of the government is given by

$$
\begin{aligned}
& \max _{\bar{g}, \underline{g}, \bar{\tau}, \underline{\tau}} \quad G^{a f}=w \bar{T}-\pi(\lambda \underline{g}+(1-\lambda) \bar{g})-s+(1-\pi) U(s) \\
& +\pi\left[\lambda H\left(s+a^{f}(\underline{\tau}, \underline{\beta})-\underline{\tau}+\underline{g}\right)+(1-\lambda) H\left(s+a^{f}(\bar{\tau}, \bar{\beta})-\bar{\tau}+\bar{g}\right)\right] \\
& \text { s.t. } \quad a^{f}(\underline{\tau}, \underline{\beta})-\underline{\tau}+\underline{g} \geq a^{f}(\bar{\tau}, \underline{\beta})-\bar{\tau}+\bar{g}, \\
& a^{f}(\bar{\tau}, \bar{\beta})-\bar{\tau}+\bar{g} \geq a^{f}(\underline{\tau}, \bar{\beta})-\underline{\tau}+\underline{g},
\end{aligned}
$$

where we added the relevant $I C$ constraints to (18). We shall assume that (A.6), the constraint from the low-cost type to the high-cost type is binding. This constraint is effectively violated at the full information solution characterized in Section 4.1. To see this recall that this solution implies

$$
a^{f}(\bar{\tau}, \bar{\beta})-\bar{\tau}+\bar{g}=a^{f}(\underline{\tau}, \underline{\beta})-\underline{\tau}+\underline{g}<a^{f}(\underline{\tau}, \bar{\beta})-\underline{\tau}+\underline{g},
$$


so that

$$
\bar{g}<\underline{g}+\bar{\tau}-\underline{\tau}+a^{f}(\underline{\tau}, \bar{\beta})-a^{f}(\bar{\tau}, \bar{\beta}),
$$

which violates condition (A.6). Substituting the incentive constraint into $G^{\text {af }}$ the government's problem can then be rewritten as

$$
\begin{aligned}
\max _{\underline{g}, \bar{\tau}, \underline{\tau}} \quad G^{a f}= & w \bar{T}-\pi\left(\underline{g}+(1-\lambda)\left(\bar{\tau}-\underline{\tau}+a^{f}(\underline{\tau}, \bar{\beta})-a^{f}(\bar{\tau}, \bar{\beta})\right)-s+(1-\pi) U(s)\right. \\
& +\pi \lambda H\left(s+a^{f}(\underline{\tau}, \underline{\beta})-\underline{\tau}+\underline{g}\right)+\pi(1-\lambda) H\left(s+a^{f}(\underline{\tau}, \bar{\beta})-\underline{\tau}+\underline{g}\right) .
\end{aligned}
$$

The FOCs are given by

$$
\begin{aligned}
\frac{\partial G^{a f}}{\partial \underline{g}} & =-\pi+\pi\left[\lambda H^{\prime}(\underline{m})+(1-\lambda) H^{\prime}(\bar{m})\right]=0, \\
\frac{\partial G^{a f}}{\partial \bar{\tau}} & =\pi(1-\lambda)\left[\frac{\partial a^{f}(\bar{\tau}, \bar{\beta})}{\partial \tau}-1\right]=0, \\
\frac{\partial G^{a f}}{\partial \underline{\tau}} & =\pi \lambda H^{\prime}(\underline{m})\left[\frac{\partial a^{f}(\underline{\tau}, \underline{\beta})}{\partial \tau}-1\right]+\pi(1-\lambda)\left(H^{\prime}(\bar{m})-1\right)\left[\frac{\partial a^{f}(\underline{\tau}, \bar{\beta})}{\partial \tau}-1\right]=0 .
\end{aligned}
$$

Recall that $a^{f}(\underline{\tau}, \bar{\beta})>a^{f}(\underline{\tau}, \underline{\beta})$, which implies $\bar{m}=s+a^{f}(\underline{\tau}, \bar{\beta})-\underline{\tau}+\underline{g}>\underline{m}=s+$ $a^{f}(\underline{\tau}, \underline{\beta})-\underline{\tau}+\underline{g}$. Hence, condition (A.7) implies that $H^{\prime}(\bar{m})<1$. Furthermore, using our assumption on $a^{f}$ along with (20), which defines $\underline{\tau}^{f f}$, we have

$$
\frac{\partial a^{f}\left(\underline{\tau}^{f f}, \bar{\beta}\right)}{\partial \tau}>\frac{\partial a^{f}\left(\underline{\tau}^{f f}, \underline{\beta}\right)}{\partial \tau}=1,
$$

so that at $\underline{\tau}^{f f}$ the first term on the RHS of (A.9) is zero while the second term is negative, which in turn implies $\underline{\tau}^{a f}<\underline{\tau}^{f f}$ (from the SOC). This is not surprising. In order to relax the IC constraint, the optimal policy distorts $\underline{\tau}$ downwards, which leads to a downward distortion on $\underline{a}$. Conversely, $\bar{\tau}$ and $\bar{a}$ are not distorted; condition (A.8) is identical to its full information counterpart (19).

Combining (A.7) with (21), the first-order condition for parents' saving, yields

$$
U^{\prime}(s)=\left[\lambda H^{\prime}(\underline{m})+(1-\lambda) H^{\prime}(\bar{m})\right]=1
$$


As in the case with uniform transfers, the optimal LTC policy implies full insurance against dependence but, under asymmetric information, it is not possible to provide insurance against the risk of having high-cost children. 


\title{
Long-term care policy with nonlinear strategic bequests*
}

\author{
Chiara Canta \\ Department of Economics, Finance and Law \\ Toulouse Business School \\ 31068 Toulouse, France \\ Helmuth Cremer \\ Toulouse School of Economics, \\ University of Toulouse Capitole \\ 31015 Toulouse, France
}

January 2017, revised Septembre 2017

*Financial support from the Chaire "Marché des risques et creation de valeur" of the FdR/SCOR is gratefully acknowledged. This paper has been presented at the EHEW 2017 in Oslo, the LAGV conference 2017 in Aix-en-Provence, and in seminars at the Universidad Autònoma de Barcelona and the Frisch Center in Oslo. We thank all the participants and particularly Marcos Vera-Hernández for their comments. 


\begin{abstract}
We study the design of long-term care (LTC) policy when children differ in their cost of providing informal care. Parents do not observe this cost, but they can commit to a "bequests rule" specifying a transfer conditional on the level of informal care. Care provided by high-cost children is distorted downwards in order to minimize the rent of low-cost ones. Social LTC insurance is designed to maximize a weighted sum of parents' and children's utility. The optimal uniform public LTC provision strikes a balance between insurance and children's utility. Under decreasing absolute risk aversion less than full insurance is provided to mitigate the distortion on informal care which reduces children's rents. A nonuniform policy conditioning LTC benefits on bequests provides full insurance even against the risk of having children with a high cost of providing care. Quite surprisingly the level of informal care induced by the optimal (uniform or nonuniform) policy always increases in the children's' welfare weight.
\end{abstract}

JEL classification: H2, H5, I13, J14.

Keywords: Long-term care, informal care, strategic bequests, asymmetric information. 


\section{Introduction}

Old age dependence and the need for long-term care (LTC) it brings about represents a major societal challenge in most developed countries. Due to population ageing the number of dependent elderly with cognitive and physical impairments will increase dramatically during the decades to come (Cremer et al., 2012). Dependence represents a significant financial risk of which only a small part is typically covered by social insurance. Private insurance markets are also thin. Instead, individuals rely on their savings or on informal care provided by family members. Currently the family is the main provider and informal care represents roughly $2 / 3$ of total care (Norton, 2000). Informal provision has no direct bearing on public finances, but it is not available to everyone. Whether this solidarity is sustainable at its current level is an important question. Sources of concerns are numerous. The drastic change in family values, the increasing number of childless households, the mobility of children, the increasing labor participation of women are as many factors explaining why the number of dependent elderly who cannot count on family solidarity, at least not for the full amount of care they need, is increasing. Furthermore, it is not clear if the important role played by informal care is desirable. Its real costs are often "hidden". In particular, it may indeed impose a significant burden on the caregivers which is both financial and psychological. ${ }^{1}$

In a nutshell, the current situation is inefficient as it leaves some elderly without proper care and often imposes a considerable burden on caregivers. This market failure creates a potential role of public intervention through social LTC provision or insurance. However, the public LTC policy will interact with informal care, and more generally with the exchanges within the family; consequently, policy design has to account for the induced changes in transfers within the family.

Informal care can be motivated by some form of altruism, result from implicit exchanges or be "imposed" by social norms. Knowing the foundation of informal care is

\footnotetext{
${ }^{1}$ On the costs borne by caregivers, see Colombo et al. (2011) or Coe and Van Houtven (2009).
} 
very important in order to predict how family assistance will react to the emergence of private or public schemes of LTC insurance.

In this paper we consider a setting where children can provide informal care to dependent parents, and intergenerational exchanges are based on a care vs. bequest (or gift) exchange. Children differ in their preferences, which determine their cost (disutility) of providing informal care. This heterogeneity reflects the fact that the level of informal care that can be obtained from the family in case of dependence depends on a number of factors such as the presence of children, their proximity, working situations, marital status, and the strength of family bonds.

Our framework is inspired by the strategic bequest approach, ${ }^{2}$ but it differs from the conventional model in a crucial way in that parents do not perfectly know their children's preferences and their cost of providing informal care. Like in the conventional model we assume that parents can commit to a bequest rule specifying a transfer, gift or bequests, conditional on the level of informal care provided. However, because of the asymmetry of information, this does no longer allow them to extract the full surplus generated by the exchange from their children. Even though parents can use nonlinear rules to screen for the children's cost parameter, they will have to leave a positive rent to some of the children.

In the laissez-faire, the help provided by high-cost children is distorted downwards in order to mitigate the rent enjoyed by the low-cost ones. Parents are not insured against the risk of dependence, nor against the risk that their children have a high cost of providing care.

We then introduce social LTC in this setting. It is designed to maximize a weighted sum of parent's and children's utilities. In other words we explicitly account for the wellbeing of caregivers. This further differentiates our paper from most of the literature, which has to a large extent concentrated on parents' welfare. ${ }^{3}$ To concentrate on LTC

\footnotetext{
${ }^{2}$ See for instance Kotlikoff and Spivak (1981), Bernheim et al. (1985).

${ }^{3}$ Barigozzi et al. (2017) also account for the welfare of caregivers, but consider a different type of
} 
policies we assume throughout the paper that the government cannot make any direct transfers to the children.

In the first part of the paper we consider a uniform social LTC policy. It provides to all dependent individuals a given LTC transfer, which is financed by a uniform lumpsum tax. We show that this policy affects distortions of informal care and thus the distribution of rents between parents and children. The optimal policy then involves a tradeoff between providing insurance to parents and enhancing the utility of the caregivers. In the absence of informal care, social LTC insurance should fully insure the risk of dependence. With informal care and strategic bequests, the optimal public LTC policy depends on the attitude towards risk of parents. Under DARA (decreasing absolute risk aversion) preferences, public LTC insurance exacerbates the distortion of informal care. Better insurance coverage makes dependent parents less reliant on informal care, so that distorting down informal care is not too costly for them. Consequently, under DARA, the optimal public LTC policy provides less than full insurance in order to mitigate the distortion of informal care and the reduction in (low-cost) children's utility it brings about. The opposite is true under IARA (increasing absolute risk aversion) preferences, in which case the government should provide more than full insurance against the risk of dependence in order to minimize distortions.

In the second part of the paper we consider nonlinear policies, where transfers form parents to children are publicly observable and LTC benefits can be conditioned on bequests (or gifts). The LTC policy can then screen for the children's cost parameter, even when the level of informal care is observable only to parents. The underlying problem presents methodological challenges because we have to deal with a "nested" principal-agent problem. While the policy can screen for the children's cost of providing care, this is done only indirectly via the parents. The latter do not observe their child's cost of providing care either but since they observe informal care, they have superior family exchanges based on cooperative bargaining. 
information.

We show that, while with a uniform policy the results crucially depend on parents' risk aversion, this is no longer true when the policy is restricted by informational considerations only and can be nonuniform. In that case the available policy instruments are sufficiently powerful to ensure that parents are always fully insured, even against the risk of having high-cost children. And since they are fully insured, risk aversion no longer matters. Even more strikingly, the tradeoff between the provision of insurance to parents and the concern for the welfare of the caregivers which drives the results for a uniform policy is not longer relevant when nonuniform policies are considered.

One surprising result regarding the impact of social LTC insurance on informal care emerges in both cases. In most of the literature on LTC, crowding out of informal care by public care is considered a serious problem. It makes the provision of social LTC insurance more expensive and possibly even ineffective, when there is full crowding out. If one accounts for the wellbeing of caregivers the impact of crowding out is more complex. One might conjecture that is "bad" for parents, but "good" for children, since it reduces the cost implied by care provision. However, this conjecture does not stand under closer scrutiny, and is proven wrong or misleading by our results. This is because in our setting children are paid for their care through gifts or bequests. Under full information, when care decreases, their compensation is reduced to keep their utility constant. Crowding out is then irrelevant. Under asymmetric information, on the other hand, crowding out will reduce the rents of some of the children and thus effectively decrease their utility, while it increases parents' welfare. Consequently, the concern for the wellbeing of caregivers does not imply that LTC policy should aim at reducing informal care.

The paper is organized as follows. We present the model in Section 2. We study the uniform and the nonlinear LTC policies, in Section 3 and 4, respectively. In Section 5 we provide some concluding remarks. 


\section{Model}

Consider a generation of identical parents. When old they face the risk of being dependent with probability $\pi$, while they are independent and healthy with probability $(1-\pi)$. When young they each have one child, they earn an exogenous labor income of $w \bar{T}$ of which they save $s$. They have preferences over consumption when young, $c \geq 0$, consumption when old and healthy, $d \geq 0$, and consumption, including LTC services, when old and dependent, $m \geq 0$. Their preferences are quasilinear in consumption when young. Risk aversion is introduced through the concavity of second period state dependent utilities. The parents' expected utility is given by

$$
E U=w \bar{T}-s+(1-\pi) U(s)+\pi E[H(m)]
$$

with $m=s+a-\tau(a)$, where $a \geq 0$ is informal care provided by children, while $\tau(a)$ is a transfer (bequest or gift) from parents to children. One can think of $m$ as total care, that is informal care $a$ plus formal care $s-\tau(a)$, the price of which is normalized to one. We assume that the transfer can be conditioned on informal care and assume that parents can commit to this bequest rule. This is in line with the strategic bequest literature. However, our model differs from the traditional literature on exchange based intergenerational transfers in that we assume that parents may not perfectly observe their child's preferences and in particular their cost of providing care. The children's heterogeneity in cost is represented by a parameter $\beta$ which is not publicly observable, including to parents. Assume that $\beta$ is distributed over $\{\underline{\beta}, \bar{\beta}\}$. In other words, $\beta$ can only take two values. The low one $\beta$ occurs with probability $\lambda \in] 0,1[$, while the high level $\bar{\beta}$ occurs with probability $(1-\lambda)$.

Children's cost of providing care $a$ to their parents is given by $v(a, \beta)$, where $0 \leq$ $a \leq a^{\max }$, with $v_{a}>0, v_{\beta}<0, v_{a a}>0, v_{a \beta}<0$, where subscripts denote partial derivatives. ${ }^{4}$ The cost is increasing and convex in the level of informal care. It decreases

\footnotetext{
${ }^{4}$ The assumption that there is an upper bound on informal care is quite natural. Furthermore it is
} 
in $\beta$, which amounts to saying that $\bar{\beta}$ is the "good type" for whom providing care is less costly. Furthermore $v_{a \beta}<0$ implies that the marginal cost of informal care also decreases with $\beta$.

The children's utility from helping their parents in case of dependence is

$$
U_{k}=c_{k}-v(a, \beta) \geq 0
$$

where children's consumption $c_{k}=\tau(a)$, that is the transfer from their parents.

Children choose $a$ to maximize $U_{k}$

$$
\tau^{\prime}(a)=v_{a}(a, \beta)
$$

and the solution to this problem is denoted $a(\beta)$. Observe that $a$ also depends on $\tau(\cdot)$, exactly as labor supply depends on the tax function in a Mirrleesian-type optimal income tax problem.

Anticipating their children's behavior but not observing $\beta$, parents choose $s$ and $\tau(a)$ to maximize their expected utility given by

$$
E U=w \bar{T}-s+(1-\pi) U(s)+\pi E_{\beta}[H(s+a(\beta)-\tau(a(\beta))] .
$$

To solve this problem we consider the equivalent mechanism design problem where parents parents choose $a(\beta)$ and $\tau(\beta)$ to maximize

$$
E U=w \bar{T}-s+(1-\pi) U(s)+\pi E_{\beta}[H(s+a(\beta)-\tau(\beta)]
$$

subject to the relevant participation constraints, as well as the incentive constraints. These constraints which come about when the parents do not observe $\beta$ will be stated and explained below.

Within this framework, we study the public provision of LTC benefits financed by a lump-sum tax on parents' first-period consumption. In addition, intergenerational convenient for technical reasons. 
transfers $\tau$ may be taxed or subsidized. The policy is determined to maximize social welfare which is given by a weighted sum of parents' and children's expected utilities. Parents' weight is $\alpha \in(0,1)$, while children's utility is weighted by $1-\alpha$.

The timing is as follows. The LTC policy is decided upon in stage 1, before parents and children make their decisions. In stage 2, parents choose their level of savings $s$ and commit to the bequest rule $\tau(a)$. In stage 3 , the dependence status of the parents is realized and children choose $a$ according to (1).

While we concentrate on the asymmetric information case, we start by considering the full information benchmark, that is the solution parents can achieve when they observe their children's cost of providing care. We also characterize the optimal LTC policy for this benchmark.

\section{Uniform LTC benefit}

In this section, we restrict the policy to a uniform transfer $g$ financed by a lump-sum tax. In other words, we consider universal public provision (or subsidization) of LTC. Such a policy is clearly suboptimal if the government can condition transfers on bequests. ${ }^{5}$ However, it is relevant in practice, since the government may not be able to observe bequests. Furthermore, even if the government was able to observe bequests, it may be politically infeasible to condition $g$ on $\tau$. In this section, we first characterize the optimal policy when parents can observe their children's type. We then turn to the asymmetric information case.

\subsection{Full information benchmark}

Parent's choose $s$ and commit to a bequest rule ex ante, that is before the state of health and $\beta$ are realized and observed. Since $\beta$ takes only two values, it is convenient

\footnotetext{
${ }^{5}$ In Section 4 we will consider the case where $g$ can be conditioned on bequests, so that the government can screen for different levels of $\beta$.
} 
to introduce the following notation

$$
\begin{array}{cll}
\tau(\bar{\beta})=\bar{\tau} & ; & \tau(\underline{\beta})=\underline{\tau}, \\
a(\bar{\beta})=\bar{a} & ; & a(\underline{\beta})=\underline{a}, \\
m(\bar{\beta})=\bar{m} & ; & \tau(\underline{\beta})=\underline{m} .
\end{array}
$$

Using this notation the parent's problem can be written as

$$
\begin{array}{rl}
\max _{\bar{a}, \underline{a}, \bar{\tau}, \underline{\tau}, s} & w \bar{T}-\pi g-s+(1-\pi) U(s)+\pi[\lambda H(s+\underline{a}-\underline{\tau}+g)+(1-\lambda) H(s+\bar{a}-\bar{\tau}+g)] \\
\text { s.t. } & \underline{\tau}-v(\underline{a}, \underline{\beta}) \geq 0 \\
& \bar{\tau}-v(\bar{a}, \bar{\beta}) \geq 0 .
\end{array}
$$

where $\pi g$ is the lump-sum tax levied to finance the expected cost of social LTC transfers. Conditions (2) and (3) represent the children's participation constraint. While children take the bequest rule $\tau(a)$ as given, they have the option not to exchange with their parents: in this case there will be no care and no transfer and chilrden's utility is an exogenously given constant which without loss of generality is normalized to zero.

Under full information, the parent can extract all the surplus, and both participation constraints are binding. ${ }^{6}$ Then, substituting for $\underline{\tau}$ and $\bar{\tau}$ from (2) and (3) the parent's problem can be rewritten as

$$
\begin{aligned}
\max _{\bar{a}, \underline{a}, s} \quad P^{f}= & w \bar{T}-\pi g-s+(1-\pi) U(s)+\pi[\lambda H(s+\underline{a}-v(\underline{a}, \underline{\beta})+g) \\
& +(1-\lambda) H(s+\bar{a}-v(\bar{a}, \bar{\beta})+g)] .
\end{aligned}
$$

The first-order conditions (FOC) with respect to the remaining choice variables are

\footnotetext{
${ }^{6}$ When a participation constraint is not binding parents can increase the corresponding $a$ and/or decrease $\tau$, thereby increasing their expected utility.
} 
given by

$$
\begin{aligned}
\frac{\partial P^{f}}{\partial \bar{a}} & =(1-\lambda) H^{\prime}(\bar{m})\left[1-v_{a}(\bar{a}, \bar{\beta})\right]=0, \\
\frac{\partial P^{f}}{\partial \underline{a}} & =\lambda H^{\prime}(\underline{m})\left[1-v_{a}(\underline{a}, \underline{\beta})\right]=0, \\
\frac{\partial P^{f}}{\partial s} & =-1+(1-\pi) U^{\prime}(s)+\pi\left[\lambda H^{\prime}(\underline{m})+(1-\lambda) H^{\prime}(\bar{m})\right]=0 .
\end{aligned}
$$

The first two conditions imply

$$
1=v_{a}(\bar{a}, \bar{\beta})=v_{a}(\underline{a}, \underline{\beta}),
$$

which is quite intuitive. Under full information parents have to compensate children exactly for their utility cost of informal care. Consequently, they equalize marginal costs to marginal benefits, which are equal to one. Not surprisingly, this implies $\bar{a}>\underline{a}$ and $\bar{m}>\underline{m}$ : low-cost children provide more informal care and their parents enjoy a larger amount of total care, $m$, in case of dependence. To decentralize this solution parents must then choose $\tau$ so that $\tau^{\prime}(\bar{a})=\tau^{\prime}(\underline{a})=1$. Observe that neither $\bar{a}$ nor $\underline{a}$ depend on $g$. Consequently, a uniform $g$ can never achieve full insurance for the risk associated with the uncertainty of $\beta$; parents with low-cost children are always better off.

We now turn to the government's problem, which is given by

$$
\begin{aligned}
\max _{g} \quad G^{f}= & w \bar{T}-\pi g-s+(1-\pi) U(s)+\pi[\lambda H(s+\underline{a}-v(\underline{a}, \underline{\beta})+g) \\
& +(1-\lambda) H(s+\bar{a}-v(\bar{a}, \bar{\beta})+g)]
\end{aligned}
$$

where $s, \underline{a}$ and $\bar{a}$ are the solutions to the parents' problem for any given $g$. Since the parents have full information, the children's utility will be zero no matter what. Consequently, the relative weight of children in social welfare is of no relevance and we can just as well neglect this term in the welfare function.

Using the envelope theorem (for the induced effect on $s$ ) and recalling that the levels 
of $a$ do not depend on $g$ we have ${ }^{7}$

$$
\frac{\partial G^{f}}{\partial g}=-\pi+\pi\left[\lambda H^{\prime}(\underline{m})+(1-\lambda) H^{\prime}(\bar{m})\right]=0 .
$$

Not surprisingly, this condition equalizes marginal costs and benefits of $g$. Combining this condition with the parent's FOC with respect to $s$ in (4) yields

$$
U^{\prime}(s)=\left[\lambda H^{\prime}(\underline{m})+(1-\lambda) H^{\prime}(\bar{m})\right]=E_{\beta}\left[H^{\prime}(m)\right]=1 .
$$

This condition states that the three possible uses of (first-period) income, direct consumption $c$, deferred consumption $s$, and LTC insurance, $g$ must have the same marginal expected utility.

\subsection{Asymmetric information}

Except for the policy design, the previous section has presented a rather standard strategic bequest model. Parents have all the bargaining power and have full information about their children's cost of providing care. We now turn to the more interesting case where parents do not know their children's type. We then have to add two incentive constraints to the parents' problem. The objective function does not change and the participation constraints continue of course to apply. The parents' problem can then be stated as follows

$$
\begin{aligned}
& \max _{\bar{a}, \underline{a}, \bar{\tau}, \underline{\tau}, s} \quad P^{a s}=w \bar{T}-\pi g-s+(1-\pi) U(s)+\pi[\lambda H(s+\underline{a}-\underline{\tau}+g) \\
& +(1-\lambda) H(s+\bar{a}-\bar{\tau}+g)] \\
& \text { s.t. } \quad \underline{\tau}-v(\underline{a}, \underline{\beta}) \geq 0, \\
& \bar{\tau}-v(\bar{a}, \bar{\beta}) \geq 0, \\
& \bar{\tau}-v(\bar{a}, \bar{\beta}) \geq \underline{\tau}-v(\underline{a}, \bar{\beta}), \\
& \underline{\tau}-v(\underline{a}, \underline{\beta}) \geq \bar{\tau}-v(\bar{a}, \underline{\beta}) .
\end{aligned}
$$

\footnotetext{
${ }^{7}$ We assume that the second order condition holds.
} 
This is a rather standard mechanism design problem and one can easily show that we obtain the "usual" pattern of binding incentive and participation constraints. To be precise, the participation constraint of high-cost children is binding so that

$$
\underline{\tau}=v(\underline{a}, \underline{\beta}) .
$$

Furthermore, the incentive constraint of $\bar{\beta}$, the low-cost type, is binding. Using (9) this condition can be written as

$$
\bar{\tau}=v(\bar{a}, \bar{\beta})+[v(\underline{a}, \underline{\beta})-v(\underline{a}, \bar{\beta})],
$$

where the term in brackets on the RHS represents the rent of type $\bar{\beta}$. In words, because low-cost children can mimic high-cost ones and provide $\underline{a}$ at a lower cost, they have to receive a transfer exceeding the cost of the care they provide. This is particularly interesting from our perspective because it implies that the utility of the caregivers is no longer exogenously given. Some of them now receive a transfers which puts them above their reservation utility level and this transfer may depend on the LTC policy.

Substituting for the transfers from (9) and (10) into the objective function, the parents' problem can then be rewritten as

$$
\begin{aligned}
\max _{\bar{a}, \underline{a}, s} \quad P^{a s}= & w \bar{T}-\pi g-s+(1-\pi) U(s)+\pi[\lambda H(s+\underline{a}-v(\underline{a}, \underline{\beta})+g) \\
& +(1-\lambda) H(s+\bar{a}-v(\bar{a}, \bar{\beta})-v(\underline{a}, \underline{\beta})+v(\underline{a}, \bar{\beta})+g)] .
\end{aligned}
$$

The first-order conditions are given by

$$
\begin{aligned}
& \frac{\partial P^{a s}}{\partial \bar{a}}=(1-\lambda) H^{\prime}(\bar{m})\left[1-v_{a}(\bar{a}, \bar{\beta})\right]=0, \\
& \frac{\partial P^{a s}}{\partial \underline{a}}=\pi\left\{\lambda H^{\prime}(\underline{m})\left[1-v_{a}(\underline{a}, \underline{\beta})\right]-(1-\lambda) H^{\prime}(\bar{m})\left[v_{a}(\underline{a}, \underline{\beta})-v_{a}(\underline{a}, \bar{\beta})\right]\right\}=0, \\
& \frac{\partial P^{a s}}{\partial s}=-1+(1-\pi) U^{\prime}(s)+\pi\left[\lambda H^{\prime}(\underline{m})+(1-\lambda) H^{\prime}(\bar{m})\right]=0 .
\end{aligned}
$$

From equation (12) we obtain $1=v_{a}(\bar{a}, \bar{\beta})$, which is the full information condition for $\bar{\beta}$, the low-cost type; see equation (5). This is the traditional no distortion at the top 
result that, given the quasi-linearity of the utility function, does not only apply to the rule, but also to the actual level of care $\bar{a}$ which is the same as in the full information solution, and continues to be independent of $g$.

Turning to $\underline{a}$, rearranging (13) yields

$$
\left[1-v_{a}(\underline{a}, \underline{\beta})\right]=\frac{(1-\lambda) H^{\prime}(\bar{m})}{\lambda H^{\prime}(\underline{m})}\left[v_{a}(\underline{a}, \underline{\beta})-v_{a}(\underline{a}, \bar{\beta})\right]>0,
$$

where we have used $v_{a \beta}<0$, which implies $\Delta v_{a}=v_{a}(\underline{a}, \underline{\beta})-v_{a}(\underline{a}, \bar{\beta})>0$. Consequently we have $v_{a}(\underline{a}, \underline{\beta})<1$ implying a downward distortion for $\underline{a}$. Intuitively, $\Delta v_{a}>0$ accounts for the fact that the rent of the low-cost type increases with $\underline{a}$. The downward distortion allows parents to mitigate this rent. Equation (15) also implies that $\underline{a}$ depends on $g$, as well as the bequests left to both types, according to (9) and (10). For $g=0$, the solution to the parents' problem yields the laissez-faire allocation.

We now turn to the government's problem. Since the parents no longer have full information, the low-cost children now have a positive utility level. Moreover, their utility is affected by the LTC policy via its impact on the parents' optimization problem. Consequently, the relative weight of children in social welfare is now relevant. When this weight is strictly positive, the LTC policy strikes a balance between providing insurance coverage to parents and the concern for the wellbeing of the caregivers.

The government's problem is given by

$$
\begin{aligned}
\max _{g} \quad G^{a s}= & \alpha\{w \bar{T}-\pi g-s+(1-\pi) U(s)+\pi[\lambda H(s+\underline{a}-v(\underline{a}, \underline{\beta})+g) \\
& +(1-\lambda) H(s+\bar{a}-v(\bar{a}, \bar{\beta})-[v(\underline{a}, \underline{\beta})-v(\underline{a}, \bar{\beta})]+g)]\} \\
& +(1-\alpha) \pi(1-\lambda)[v(\underline{a}, \underline{\beta})-v(\underline{a}, \bar{\beta})],
\end{aligned}
$$

where $\bar{a}, \underline{a}$ and $s$ are determined by the solution to the parents' problem. Observe that parents' utility is expressed as the solution to the reformulated problem (11) which is an unconstrained optimization where the relevant IC and participation constraints have been substituted into the objective function. 
Using the envelope theorem according to which we can neglect the derivatives of parents' utility with respect to $\underline{a}, \bar{a}$, and $s$, the FOC is given by ${ }^{8}$

$$
\begin{aligned}
\frac{\partial G^{a s}}{\partial g}=\alpha & \left.\alpha-\pi+\pi\left[\lambda H^{\prime}(\underline{m})+(1-\lambda) H^{\prime}(\bar{m})\right]\right\} \\
& +\pi(1-\lambda)(1-\alpha)\left[v_{a}(\underline{a}, \underline{\beta})-v_{a}(\underline{a}, \bar{\beta})\right] \frac{\partial \underline{a},}{\partial g}=0 .
\end{aligned}
$$

Recall that the term $\Delta v_{a}=v_{a}(\underline{a}, \underline{\beta})-v_{a}(\underline{a}, \bar{\beta})$ is positive. Observe that the children's utility does not directly depend on $s$ and that $\bar{a}$ does not depend on $g$, nor does the high-costs children's utility, which is always equal to zero, the exogenous reservation utility. Consequently, the only behavioral response to the LTC policy that is relevant in (17) is $\partial \underline{a}, / \partial g$ which measures how $g$ affects the level of care provided by the high-cost children. The sign of this expression will in turn determine how the level of care affects the caregivers' utility.

Consider first the case where the caregivers' utility is not included in social welfare, that is $\alpha=1$. Using the parent's FOC, expression (17) can be written as

$$
U^{\prime}(s)=\left[\lambda H^{\prime}(\underline{m})+(1-\lambda) H^{\prime}(\bar{m})\right]=1 .
$$

This is the same rule as the one under full information that was given by (7). In both cases we have $U^{\prime}(s)=1$ so that the level of $s$ is also the same. However, the levels of $m$ will differ from the full information solution, which in turn implies that the level of $g$ will also in general be different, even though the rule is the same.

We use superscripts $f$ and as to refer to the solutions to the full information and asymmetric information problems, specified by (6) and (16) respectively. Suppose that $g^{a s}=g^{f}$. Then, $\lambda H^{\prime}\left(\underline{m}^{a s}\right)+(1-\lambda) H^{\prime}\left(\bar{m}^{a s}\right)>1$, because $\underline{m}^{a s}<\underline{m}^{f}$ (as $\underline{a}$ is distorted downward) and $\bar{m}^{a s}<\bar{m}^{f}$ (as low-cost children receive a positive rent); consequently we must have $g^{a s}>g^{f}$ : the optimal level of LTC benefits is larger under asymmetric information than under full information. Intuitively, $g$ is higher to compensate in part for the downward distortion in $a$ that parents create to mitigate children's rents.

\footnotetext{
${ }^{8}$ We assume that the second order condition holds.
} 
Let us now turn to the case where $\alpha<1$, which includes the utilitarian case where $\alpha=1 / 2$. In this case $g$ is no longer solely determined to provide insurance to parents. The optimal LTC policy also accounts for the impact of $g$ on informal care and thus on children's utility (rents). Roughly speaking, when $\partial \underline{a} / \partial g>0$ one can expect that the effect described for $\alpha=1$ is reinforced by the effect of $g$ on children's rents. Since rents increase in $\underline{a}$, increasing $g$ increases rents. In this case we have $g^{a s}>g^{f}$. Conversely, when $\partial \underline{a} / \partial g<0$, the two effects go in opposite directions. Either way this discussion shows that as soon as $\alpha<1$ the results will crucially depend on the sign of $\partial \underline{a} / \partial g$. The study of this sign requires a closer look at the comparative statics of the parents' problem under asymmetric information. The following lemma is established in Appendix A.1.

Lemma 1 When the parents' utility in case of dependence $H(m)$ exhibits DARA we have $\partial \underline{a} / \partial g<0$; when $H(m)$ exhibits IARA we have $\partial \underline{a} / \partial g>0$.

This lemma shows that the effect of $g$ (via the parents' problem) on the level of care provided by the high-cost children depends on the parents' attitude towards risk. Intuitively our results can be understood as follows. With DARA, as $g$ increases, parents become less risk averse. Then, reducing $m$ in the bad state of nature becomes less costly for them, and distorting $\underline{a}$ downwards becomes more attractive. The case with IARA is exactly symmetrical. Note that empirically DARA appears to receive more support (Friend and Blume, 1975).

Using equation (17) and Lemma 1 we can study the effect of $\alpha$ on $g^{a s}(\alpha)$. For instance, we can compare the utilitarian level $g^{a s}(1 / 2)$ with $g^{a s}(1)$, the level achieved when children are not accounted for in the social welfare function. With DARA we know from Lemma 1 that $\underline{a}$ decreases as $g$ increases, which in turn implies that the utility of the low-cost child decreases; recall that $\Delta v_{a}>0$. Consequently equation (17) evaluated at $g^{a s}(1)$ is negative so that $g^{a s}(1 / 2)<g^{a s}(1)$. Under IARA these effects are reversed and we obtain $g^{a s}(1 / 2)>g^{a s}(1)$. This result also goes through for intermediate levels 
of $\alpha$. Totally differentiating (17) and rearranging yields

$$
\frac{\partial g}{\partial \alpha}=\frac{\frac{(1-\lambda)}{\alpha}\left[v_{a}(\underline{a}, \underline{\beta})-v_{a}(\underline{a}, \bar{\beta})\right] \frac{\partial \underline{a}}{\partial g}}{S O C},
$$

which has the opposite sign of $\partial \underline{a} / \partial g$. Accordingly, under DARA $g$ increases in $\alpha$, while it decreases as $\alpha$ increases under IARA.

Using the FOC of the parents with respect to $s$, (14), equation (17) implies that, whenever $\alpha<1$

$$
U^{\prime}(s)<1<E_{\beta}\left(H^{\prime}(m)\right) \quad \text { if } \quad \partial \underline{a} / \partial g<0,
$$

and

$$
U^{\prime}(s)>1>E_{\beta}\left(H^{\prime}(m)\right) \quad \text { if } \quad \partial \underline{a} / \partial g>0 .
$$

Intuitively, the utility of dependent parents is distorted down with respect to the full information case under DARA. In this case, providing full insurance against the risk of dependence would push parents to cut the utility of low-cost children (by distorting down $\underline{a}$ ). From the perspective of social welfare, there is a then a tradeoff between insurance and children's utility, leading to less than full insurance. Accordingly, parents have an incentive to save more and this increases their consumption if healthy. Under IARA these effects are reversed.

The results obtained in this section are summarized in the following proposition.

Proposition 1 Consider the case where the children's cost of providing care is not observable and where public policy is restricted to a uniform LTC benefit $g$ financed by a lump-sum tax. Informal care is observable only by parents. The optimal LTC policy is such that:

(i) The risk of having children with a high cost of providing care is not fully insured;

(ii) If children's utility has no weight in social welfare, parents are fully insured against dependence. This is achieved through a uniform benefit that is larger than in the full information case. 
(iii) If the weight of children in social welfare is strictly positive, and the parent's utility in case of dependence $H(m)$ exhibits DARA, parents are less than fully insured against dependence. The uniform benefit decreases with the weight of children in social welfare.

(iv) If the weight of children in social welfare is strictly positive, and $H(m)$ exhibits IARA, parents are more than fully insured against dependence. The uniform benefit is higher than under full information and it increases with the weight of children in social welfare.

\section{Nonlinear policies}

We now consider nonlinear policies under which the level of LTC transfers $g$ can be conditioned on $\tau$, which is publicly observable. The LTC policy can then screen for the children's cost parameter $\beta$. We continue to assume that $a$ is observable only to parents. The underlying problem presents methodological challenges because we have to deal with a "nested" principal-agent problem. ${ }^{9}$ While the policy can screen for $\beta$, this is done only indirectly via the parents. The latter do not observe their child's $\beta$ either but since they observe informal care, they have superior information.

We proceed exactly like in the previous section. We start with the full information solution and then concentrate on the case where neither the parents nor the government can observe the children's type $\beta .^{10}$ Parents observe $a$ but the government does not. The policy we study consists of a menu of LTC benefits and bequests pairs. Under asymmetric information parents self-select into a benefit-bequest pair. We will also continue to assume that the government cannot make any direct transfer to children.

Observe that, while we study a mechanism design problem, the policy can be imple-

\footnotetext{
${ }^{9}$ The problem considered by Guesnerie and Laffont (1978) has a similar structure. They analyze nonlinear taxation of a monopolist that in turn uses nonlinear pricing.

${ }^{10}$ The "intermediate" case where only parents have full information is also of some interest. However, since the insight it provides is not directly related to our main results, we restrict ourselves to presenting it in an Appendix.
} 
mented by a suitably designed mix of LTC benefits and taxes. The mix depends on the precise timing and more specifically on whether $\tau$ is interpreted as a gift or a bequest. So far we have been agnostic about this because it was of no relevance. When $\tau$ is a gift and thus precedes the public transfer $g$, the solution can be implemented simply by a function $g(\tau)$ conditioning LTC benefits on $\tau$. When $\tau$ is a bequest, which by definition occurs after $g$ is consumed, we can condition LTC on a reported (planned) $\tau$, but then we have to make sure that parents stick to one of the pairs $(\underline{\tau}, \underline{g})$ or $(\bar{\tau}, \bar{g})$. In particular we have to prevent parents from picking a pair, but then leaving a larger bequest (in order to "buy" more care). This can be done by a nonlinear tax on bequest which is prohibitively large when $\tau$ deviates from the one associated with the level of public LTC consumed. In practice this means that "excess" public transfers can be recovered from an individual's bequest.

\subsection{Full information solution}

In this section, we assume that both parents and the government have full information concerning the children's types. Transfer $\tau$ are also publicly observable. However, $a$ is observed by parents only. The government sets $\bar{g}, \underline{g}, \bar{\tau}, \underline{\tau}$, anticipating the choices of the parents. Parents choose $s$ ex ante to maximize their expected utility, and set a such that $\tau-v(a, \beta)=0$; we can thus define $a^{f}(\tau, \beta)$ as the solution to this equation. We have

$$
\begin{aligned}
& \frac{\partial a^{f}}{\partial \tau}=\frac{1}{v_{a}} \\
& \frac{\partial a^{f}}{\partial \beta}=-\frac{v_{\beta}}{v_{a}}>0 .
\end{aligned}
$$

Parents also . 
The government now maximizes

$$
\begin{aligned}
\max _{\bar{g}, \underline{g}, \bar{\tau}, \underline{\tau}} \quad G^{f f}= & w \bar{T}-\pi(\lambda \underline{g}+(1-\lambda) \bar{g})-s+(1-\pi) U(s) \\
& +\pi\left[\lambda H\left(s+a^{f}(\underline{\tau}, \underline{\beta})-\underline{\tau}+\underline{g}\right)+(1-\lambda) H\left(s+a^{f}(\bar{\tau}, \bar{\beta})-\bar{\tau}+\bar{g}\right)\right] .
\end{aligned}
$$

The FOCs of the government are

$$
\begin{aligned}
& \frac{\partial G^{f f}}{\partial \bar{g}}=-\pi(1-\lambda)+\pi(1-\lambda) H^{\prime}(\bar{m})=0, \\
& \frac{\partial G^{f f}}{\partial \underline{g}}=-\pi \lambda+\pi \lambda H^{\prime}(\underline{m})=0 \\
& \frac{\partial G^{f f}}{\partial \bar{\tau}}=\pi(1-\lambda) H^{\prime}(\bar{m})\left[\frac{\partial a^{f}(\bar{\tau}, \bar{\beta})}{\partial \tau}-1\right]=0, \\
& \frac{\partial G^{f f}}{\partial \underline{\tau}}=\pi \lambda H^{\prime}(\underline{m})\left[\frac{\partial a^{f}(\underline{\tau}, \underline{\beta})}{\partial \tau}-1\right]=0,
\end{aligned}
$$

and parents choose $s$ so that

$$
\frac{\partial G^{f f}}{\partial s}=-1+(1-\pi) U^{\prime}(s)+\pi\left[\lambda H^{\prime}(\underline{m})+(1-\lambda) H^{\prime}(\bar{m})\right]=0 .
$$

Combining these equations yields

$$
H^{\prime}(\bar{m})=H^{\prime}(\underline{m})=U(s)=1,
$$

and

$$
v_{a}(\bar{a}, \bar{\beta})=v_{a}(\underline{a}, \underline{\beta})=1 .
$$

These expressions have a simple interpretation. With full information, a nonuniform LTC policy can provide full insurance not only against the risk of dependence, but also against the risk of having high-cost children. Informal care $a$ is set at the efficient levels for each type of children. We now turn to the case where neither parents nor the government observe the children's types. The "intermediate" case where only parents have full information is also of some interest. It is presented in Appendix A.2, which can be skipped without affecting the readability of the following sections. 


\subsection{Asymmetric information}

When neither the parents nor the government can observe the children's types, the government proposes a menu $((\bar{\tau}, \bar{g}),(\underline{\tau}, \underline{g}))$. The only choice left to parents is to fix the level of $a$ associated with each option. As long as $\bar{\tau}>\underline{\tau}$, parents set these levels of informal care such that the participation constraint of $\beta$ and the incentive constraint of $\bar{\beta}$ are satisfied. Formally, the levels of $a$ are defined by

$$
\underline{\tau}=v\left(\underline{a}^{a s}, \underline{\beta}\right),
$$

and

$$
\bar{\tau}=v\left(\bar{a}^{a s}, \bar{\beta}\right)+v\left(\underline{a}^{a s}, \underline{\beta}\right)-v\left(\underline{a}^{a s}, \bar{\beta}\right) .
$$

The optimal (nonuniform) LTC policy is then determined by solving the following problem ${ }^{11}$

$$
\begin{aligned}
\max _{\bar{g}, \underline{,}, \bar{\tau}, \underline{\tau}} \quad G^{a a}= & \alpha\{w \bar{T}-\pi(\lambda \underline{g}+(1-\lambda) \bar{g})-s+(1-\pi) U(s) \\
& \left.+\pi\left[\lambda H\left(s+\underline{a}^{a s}-\underline{\tau}+\underline{g}\right)+(1-\lambda) H\left(s+\bar{a}^{a s}-\bar{\tau}+\bar{g}\right)\right]\right\} \\
& +(1-\alpha) \pi(1-\lambda)\left[v\left(\underline{a}^{a s}, \underline{\beta}\right)-v\left(\underline{a}^{a s}, \bar{\beta}\right)\right] \\
\text { s.t. } \quad \underline{\tau} & =v\left(\underline{a}^{a s}, \underline{\beta}\right), \\
& \bar{\tau} \\
& =v\left(\bar{a}^{a s}, \bar{\beta}\right)+v\left(\underline{a}^{a s}, \underline{\beta}\right)-v\left(\underline{a}^{a s}, \bar{\beta}\right), \\
& \bar{\tau} \geq \underline{\tau} .
\end{aligned}
$$

To solve this problem, we will first ignore constraint (26). We will then verify ex post if the solution to the unconstrained problem fulfils this constraint. If this is the case,

${ }^{11}$ We will ignore for the moment the IC constraints of the parents, which are given by

$$
\begin{aligned}
& \lambda H\left(s+\underline{a}^{a s}-\underline{\tau}+\underline{g}\right)+(1-\lambda) H\left(s+\bar{a}^{a s}-\bar{\tau}+\bar{g}\right) \geq H\left(s+\underline{a}^{a s}-\underline{\tau}+\underline{g}\right) \\
& \lambda H\left(s+\underline{a}^{a s}-\underline{\tau}+\underline{g}\right)+(1-\lambda) H\left(s+\bar{a}^{a s}-\bar{\tau}+\bar{g}\right) \geq H\left(s+a^{a s}(\bar{\tau}, \underline{\beta})-\bar{\tau}+\bar{g}\right),
\end{aligned}
$$

where $\bar{\tau}=v\left(a^{a s}(\bar{\tau}, \underline{\beta}), \underline{\beta}\right)$. In words, since parents have private information on the level of informal care, the social planner has to ensure that they will not prefer a contract over the other, and propose a pooling contract to the children. In Footnote 12, we show that these constraints are indeed not binding. 
we can indeed ignore the constraint. If not a pooling equilibrium emerges. Substituting (24) and (25) in the objective function, the problem can be rewritten as

$$
\begin{aligned}
\max _{\bar{g}, \underline{a}, \bar{a}, \underline{a}} \quad G^{a a}= & \alpha\{w \bar{T}-\pi(\lambda \underline{g}+(1-\lambda) \bar{g})-s+(1-\pi) U(s) \\
& +\pi \lambda H(s+\underline{a}-v(\underline{a}, \underline{\beta})+\underline{g}) \\
& +\pi(1-\lambda) H(s+\bar{a}-v(\bar{a}, \bar{\beta})-v(\underline{a}, \underline{\beta})+v(\underline{a}, \bar{\beta})+\bar{g})\} \\
& +(1-\alpha) \pi(1-\lambda)[v(\underline{a}, \underline{\beta})-v(\underline{a}, \bar{\beta})] .
\end{aligned}
$$

The FOCs are given by

$$
\begin{aligned}
\frac{\partial G^{a a}}{\partial \bar{g}} & =-\pi(1-\lambda)+\pi(1-\lambda) H^{\prime}(\bar{m})=0 \\
\frac{\partial G^{a a}}{\partial \underline{g}} & =-\pi \lambda+\pi \lambda H^{\prime}(\underline{m})=0 \\
\frac{\partial G^{a a}}{\partial \bar{a}} & =\pi(1-\lambda) H^{\prime}(\bar{m})\left[1-v_{a}(\bar{a}, \bar{\beta})\right]=0 \\
\frac{\partial G^{a a}}{\partial \underline{a}} & =\pi \alpha \lambda H^{\prime}(\underline{m})\left[1-v_{a}(\underline{a}, \underline{\beta})\right]-\pi(1-\lambda)\left[\alpha H^{\prime}(\bar{m})-(1-\alpha)\right]\left[v_{a}(\underline{a}, \underline{\beta})-v_{a}(\underline{a}, \bar{\beta})\right]=0
\end{aligned}
$$

Conditions (27) and (28), combined with the parents' FOC with respect to savings imply that

$$
U^{\prime}(s)=H^{\prime}(\underline{m})=H^{\prime}(\bar{m})=1
$$

Then, we have $\underline{m}=\bar{m}$ implying that, under asymmetric information, the optimal nonuniform LTC insurance scheme provides full insurance not only against the risk of dependence, but also against the uncertainty associated with informal care. This is in stark contrast with the results obtained with a uniform policy where full insurance could not be achieved.

Informal care is set at its first best level for low-cost children, as it is shown in (29). Conversely, the optimal level of informal care provided by high-cost children is distorted. Combining (29) with (28) and (30) shows that an interior solution for $\underline{a}$ is determined 
by

$$
\lambda \alpha\left[1-v_{a}(\underline{a}, \underline{\beta})\right]-(1-\lambda)(2 \alpha-1)\left[v_{a}(\underline{a}, \underline{\beta})-v_{a}(\underline{a}, \bar{\beta})\right]=0 .
$$

Since $v_{a}$ decreases in $\beta$, the direction of the distortion depends on the sign of $(2 \alpha-1)$. If children have a lower weight than parents in the social welfare function $(\alpha>1 / 2)$, then $\underline{a}$ is distorted downward. If parents and children have the same weight, $(\alpha=1 / 2)$, there will be no distortion of informal care. Intuitively, the rent is purely a transfer in this case, since the social marginal utility of consumption is the same for children and parents. Finally, if children have a higher weight than parents, there will be an upward distortion of informal care.

The optimal LTC transfer is higher the lower the level of bequests (and the lower the level of informal care). To see this, consider the allocation characterized by (27)-(30). Since individuals are fully insured, $\underline{m}=\bar{m}$, which implies

$$
g(\bar{\tau}) \leq g(\underline{\tau}) \Longleftrightarrow \bar{a}-\bar{\tau} \geq \underline{a}-\underline{\tau} .
$$

Using the IC and PC of the children, this can be rewritten as

$$
g(\bar{\tau}) \leq g(\underline{\tau}) \Longleftrightarrow \bar{a}-v(\bar{a}, \bar{\beta}) \geq \underline{a}-v(\underline{a}, \bar{\beta}),
$$

which is always true since $\bar{a}=\arg \max _{x} x-v(x, \bar{\beta})$. Then, the optimal separating policy $g(\tau)$ implies higher transfers to the parents of high-cost children, in order to compensate them for the lower level of informal care they receive. ${ }^{12}$

The allocation characterized above is a solution to the government's problem only if it satisfies (26). Denote $\underline{a}^{*}$ and $\bar{a}^{*}$ the solutions to (31) and (29). Differentiating (31) shows that $\underline{a}^{*}$ always decreases in $\alpha$ (and thus increases in children's weight $1-\alpha$ ), irrespective of the degree of risk aversion of the parents. ${ }^{13}$ If children have no weight in

\footnotetext{
${ }^{12}$ This solution implies $\underline{m}=\bar{m}$ so that the parents' IC constraints (22) and (23) stated in Footnote 11 are satisfied.

${ }^{13}$ Differentiating (31), one obtains that the sign of $\partial \underline{a}^{*} / \partial \alpha$ is equal to the sign of

$$
\lambda\left[1-v_{a}(\underline{a}, \underline{\beta})\right]-2(1-\lambda)\left[v_{a}(\underline{a}, \underline{\beta})-v_{a}(\underline{a}, \bar{\beta})\right],
$$

which is always negative under (31) since $(2 \alpha-1) / \alpha \leq 1$ for all $\alpha \leq 1$.
} 
the social welfare function $(\alpha=1)$, then $\underline{a}$ is distorted downwards, so that $\underline{a}^{*}<\bar{a}^{*}$. If $\alpha=0$, then the LHS of (31) is always positive. In this case there is a corner solution with $\underline{a}^{*}=a^{\max } \geq \bar{a}^{*}$, where $a^{\max }$ is the maximum level of informal care that can be provided by children. Then, there exist a threshold $\widehat{\alpha}=(1-\lambda) /(2-\lambda)<1 / 2$ such that the optimal policy implies $\underline{a}=\underline{a}^{*} \leq \bar{a}^{*}$ if and only if $\alpha \geq \widehat{\alpha} \cdot{ }^{14}$ Using (24) and (25) it follows that this solution satisfies constraint (26).

When $\alpha<\widehat{\alpha}$ we have $\underline{a}^{*}>\bar{a}^{*}$, which violates constraint (26), since

$$
\underline{\tau}=v\left(\underline{a}^{*}, \underline{\beta}\right)>v\left(\bar{a}^{*}, \bar{\beta}\right)+v\left(\underline{a}^{*}, \underline{\beta}\right)-v\left(\underline{a}^{*}, \bar{\beta}\right)=\bar{\tau},
$$

whenever $\underline{a}^{*}>\bar{a}^{*}$. In this case, the optimal policy consists in a pooling contract $\left\{\tau^{p}, g^{p}\right\}$. Observe that, since $\widehat{\alpha}<1 / 2$, pooling requires that children receive a larger weight than parents in social welfare.

To complete the analysis let us now determine this pooling equilibrium. Under this contract parents set $a^{p}$ such that $\tau^{p}=v\left(a^{p}, \underline{\beta}\right)$ and the government's problem can be written as

$$
\begin{aligned}
\max _{g, a} \quad G^{a a p}= & \alpha\{w \bar{T}-\pi g-s+(1-\pi) U(s)+\pi H(s+a-v(a, \underline{\beta})+g)\} \\
& +(1-\alpha) \pi(1-\lambda)[v(a, \underline{\beta})-v(a, \bar{\beta})] .
\end{aligned}
$$

The FOCs are given by

$$
\begin{aligned}
& \frac{\partial G^{\text {aap }}}{\partial g}=-\pi+\pi H^{\prime}=0, \\
& \frac{\partial G^{\text {aap }}}{\partial a}=\pi \alpha H^{\prime}\left[1-v_{a}(a, \underline{\beta})\right]+\pi(1-\lambda)(1-\alpha)\left[v_{a}(a, \underline{\beta})-v_{a}(a, \bar{\beta})\right]=0 .
\end{aligned}
$$

Condition (32) yields $H^{\prime}=1$. This, combined with the FOC of the parents, implies $1=U^{\prime}(s)=H^{\prime}(m)$ so that we continue to have full insurance against both the risk of dependence and the risk of having a high-cost child. Furthermore, (33) can be rewritten as

$$
\left[1-v_{a}\left(a^{p}, \underline{\beta}\right)\right]+(1-\lambda) \frac{(1-\alpha)}{\alpha}\left[v_{a}\left(a^{p}, \underline{\beta}\right)-v_{a}\left(a^{p}, \bar{\beta}\right)\right]=0 .
$$

\footnotetext{
${ }^{14}$ The threshold $\widehat{\alpha}$ is the value of $\alpha$ such that (31) is satisfied for $\underline{a}=\bar{a}^{*}$.
} 
Differentiating this expression and making use of the SOC show that $\partial a^{p} / \partial \alpha<0$ so that under the pooling contract informal care continues to increase with the weight of children in the social welfare function.

The main results of this section are summarized in the following proposition.

Proposition 2 Consider the case where children's cost of providing care is not observable and where LTC benefits $g$ can be conditioned on the transfer $\tau$ paid by parents to children in exchange for informal care. Informal care is observable only to parents. The optimal LTC policy may involve a separating or a pooling contract. This policy is such that:

(i) The risk of having high-cost children is fully insured.

(ii) The average level of informal care always increases in the weight of children in the social welfare function, irrespective of the parents' degree of risk aversion.

(iii) A separating contract is optimal if and only if $\alpha \geq(1-\lambda) /(2-\lambda)<1 / 2$. It implies that:

(a) Informal care is set at its first best level for the low-cost children.

(b) The level of informal care provided by high-cost children is distorted and the direction of the distortion depends on children's weight in the welfare function. It has the same sign as $(2 \alpha-1)$ so that a downward (upward) distortion occurs when the weight of the children is lower (higher) than the weight of parents.

Note that, while with a uniform policy the results crucially depend on parents' risk aversion, this is no longer true when the policy is restricted by informational considerations only, and can be nonuniform. In that case the available policy instruments are sufficiently powerful to ensure that parents are always fully insured, even against the risk of having high-cost children. And since they are fully insured, risk aversion no 
longer matters. Even more strikingly, the tradeoff between the provision of insurance to parents and the concern for the welfare of the caregivers which drives the results for a uniform policy is no longer relevant under nonuniform policies.

\section{Conclusion}

We study the design of long-term care (LTC) policy when informal care from children to dependent parents is due to a bequest motive. Parents do not observe their children's cost of providing care, but they can commit to a bequests rule specifying a transfer conditional on the level of informal care. The social welfare function is a weighted sum of parents' and children's utility. We show that social LTC insurance affects the exchanges between parents and children and in particular the level of informal care and the distribution of rents.

The optimal uniform public LTC insurance depends on the attitude towards risk of parents. Under DARA (decreasing absolute risk aversion) preferences, public LTC insurance exacerbates the distortion of informal care. Consequently, the optimal public LTC coverage provides less than full insurance. The opposite is true under IARA (increasing absolute risk aversion) preferences. A uniform policy can never insure the risk of having a high-cost child.

A nonuniform policy that conditions LTC benefits on bequests provides full insurance even for the risk of having high-cost children. The level of informal care provided by high-cost children is distorted and the direction of the distortion depends on children's weight in the social welfare function.

Interestingly, in the uniform as well as in the nonuniform case, the higher the weight of children in the social welfare function, the higher the optimal average level of informal care. This apparently counter-intuitive result is due to the exchange motive behind family help. Under this motive, higher reliance on the family for the provision of longterm care implies higher rents for children. In our model, crowding out of family help by 
public care only affects social welfare through its (negative) effect on children's utility. A main lesson that emerges from our analysis is that in an exchange-based setting, social insurance should be designed in order to ensure that dependent elderly have to rely even on high-cost children. This ensures that low-cost children get rewarded for the informal care they provide.

Another major lesson is that, even with ex ante identical individuals, the nonuniform policy performs better and is able to provide full insurance against both underlying risks. In other words, even with identical individuals, social LTC should involve some measure of means testing and/or recover part of the benefits received by the elderly from their estate. This results is interesting because means testing is usually justified by redistribution. We have not considered redistributive motive, but they could only be expected to reinforce this result.

Throughout the paper we have remained agnostic about the exact nature of the transfer, gift or bequest, that "pays" for the care, except that we have pointed out that it affects the timing of the underlying game. From and empirical perspective, however, the gift interpretation appears to be more compelling. The literature has found some evidence that inter vivos transfers are larger for those children who provide informal care (Norton and Van Houtven, 2006). However, these estimates are often problematic because of endogeneity problems and because measurement of both informal care and financial transfers within the family is difficult. More reliable estimates require longitudinal data and a step in that direction is taken by Norton et al. (2014) who use the 1999 and 2003 waves of National Longitudinal Survey of Mature Women and show that children providing informal care are indeed more likely to receive financial transfers from their parents.

Considering bequests as payment for care is more problematic because research has shown that bequests are typically divided equally among children (Menchik, 1980; Tomes, 1981). Even when children provide unequal amounts of informal care, bequests 
tend to be divided equally (Norton and Taylor, 2005). This is true for the US but the argument is even more compelling for most European countries, where equal sharing rules are imposed by law. In any event there are also theoretical arguments that favor inter vivos transfers over bequests as payment for informal care. Gifts are more flexible and, as argued by Norton and Van Houtven (2006), "can be adjusted quickly to the amount of care, are less costly than writing a will, and can be kept secret from other family members and the public".

Our policy recommendations are made under the assumption that the provision of informal care is exchange-based. This hypothesis has received empirical support and appears to apply to certain families. Roughly speaking, “... the idea of exchange makes sense for those extended families where an older person has money and needs help, and a younger person has time and needs money", (Norton et al. 2014). But it is clearly not the only behavioral pattern that is relevant. In reality the different types of intra-family relations, based on altruism, norms or selfish exchanges, with and without commitment are likely to coexist. ${ }^{15}$ The different studies provide partial and intermediate answers which can provide valuable guidance for the design of social LTC policy, as long as interpreted with suitable care and keeping in mind the underlying assumptions.

\footnotetext{
${ }^{15}$ Alternative approaches explored for instance by Cremer and Roeder (2017) Barrigozzi et al. (2017), Canta and Pestieau (2014), Ponthière (2014).
} 


\section{References}

[1] Barigozzi, F., H. Cremer and K. Roeder, (2017), "Caregivers in the family: daughters, sons and social norms," TSE-Working Paper.

[2] Bernheim, B., A. Shleifer and L. Summers, (1985), "The strategic bequest motive", Journal of Political Economy, 93, 1045-1076.

[3] Colombo, F., A. Llena-Nozal, J. Mercier and F. Tjadens, (2011), "Help Wanted? Providing and paying for long-term care", OECD Health Policy Studies, OECD Publishing.

[4] Canta, C. and P. Pestieau, (2014), "Long-Term Care Insurance and Family Norms", BEJEAP, 14, 401-429.

[5] Cremer, H., P. Pestieau and G. Ponthière, (2012), "The economics of long-term care: A survey", Nordic Economic Policy Review, 2, 107-148.

[6] Cremer, H. and K. Roeder, (2017), "Long-term care policy with lazy rotten kids", Journal of Public Economic Theory, forthcoming.

[7] Coe, N. B. and C. H. Van Houtven, (2009), "Caring for Mom and Neglecting Yourself? The Health Effects of Caring for an Elderly Parent." Health Economics, 18, 991-1010.

[8] Friend, I. and M. Blume, (1975), "The Demand for Risky Assets", American Economic Review, 65, 900-922.

[9] Guesnerie, R. and J.-J. Laffont, (1978), "Taxing price makers", Journal of Economic Theory, 19, 423-455.

[10] Kotlikoff, L. and A. Spivak (1981) "The Family as an Incomplete Annuities Market." Journal of Political Economy, 89, 372-91. 
[11] Menchik, P. L., (1980), "Primogeniture, Equal Sharing and the U.S. Distribution of Wealth." Quarterly Journal of Economics, 94, 299-316.

[12] Norton, E., (2000), "Long term care", in A. Cuyler \& J. Newhouse (Eds.): Handbook of Health Economics, Volume 1b, chapter 17.

[13] Norton, E., H. Nicholas and S. Huang, (2014), Informal Care and Inter-vivos Transfers: Results from the National Longitudinal Survey of Mature Women, BEJEAP, $14,377-401$.

[14] Norton, E. C. and D. H. Taylor, Jr, (2005), "Equal Division of Estates and the Bequest Motive." Journal of Aging and Social Policy, 17, 63-82.

[15] Norton, E. C. and C. H. Van Houtven, (2006), "Inter-vivos Transfers and Exchange." Southern Economic Journal 73, 157-72.

[16] Ponthière, G., (2014), "Long-Term Care, Altruism and Socialization", BEJEAP, $14,429-473$.

[17] Tomes, N., (1981) "The Family, Inheritance and the Intergenerational Transmission of Inequality." Journal of Political Economy, 89, 928-58. 


\section{Appendix}

\section{A.1 Proof of Lemma 1}

Since $\bar{a}$ is independent off $g$, we can focus on the FOCs (13) and (14) to study the comparative statics with respect to $g$. Using subscripts to denote partial derivative, define

$$
H=\left[\begin{array}{ll}
P_{a a}^{a s} & P_{s a}^{a s} \\
P_{\underline{a} s}^{a s} & P_{s s}^{a s}
\end{array}\right]
$$

and

$$
D=\left[\begin{array}{c}
-P_{a g}^{a s} \\
-P_{s g}^{a s}
\end{array}\right]
$$

where

$$
\begin{aligned}
& P_{s \underline{a}}^{a s}=P_{\underline{a} s}^{a s}=\pi\left[\lambda H^{\prime \prime}(\underline{m})\left(1-v(\underline{a}, \underline{\beta})-(1-\lambda) H^{\prime \prime}(\bar{m})\left(v_{a}(\underline{a}, \underline{\beta})-v_{a}(\underline{a}, \bar{\beta})\right)\right]=\pi A,\right. \\
& P_{s s}^{a s}=(1-\pi) U^{\prime \prime}(s)+\pi\left[\lambda H^{\prime \prime}(\underline{m})+(1-\lambda) H^{\prime \prime}(\bar{m})\right], \\
& P_{\underline{a} g}^{a s}=\pi\left\{\lambda H^{\prime \prime}(\underline{m})\left[1-v_{a}(\underline{a}, \underline{\beta})\right]-(1-\lambda) H^{\prime \prime}(\bar{m})\left[v_{a}(\underline{a}, \underline{\beta})-v_{a}(\underline{a}, \bar{\beta})\right]\right\}=\pi A, \\
& P_{s g}^{a s}=\pi\left[\lambda H^{\prime \prime}(\underline{m})+(1-\lambda) H^{\prime \prime}(\bar{m})\right],
\end{aligned}
$$

and where

$$
A=\lambda H^{\prime \prime}(\underline{m})\left[1-v_{a}(\underline{a}, \underline{\beta})\right]-(1-\lambda) H^{\prime \prime}(\bar{m})\left[v_{a}(\underline{a}, \underline{\beta})-v_{a}(\underline{a}, \bar{\beta})\right] .
$$

Using Cramer's rule we obtain

$$
\frac{\partial \underline{a}}{\partial g}=\frac{\left|\begin{array}{cc}
-P_{a g}^{a s} & P_{s a}^{a s} \\
-P_{s g}^{a s} & P_{s s}^{a s}
\end{array}\right|}{|H|}
$$

where $|H|>0$ from the SOC.

Substituting from (A.1)-(A.4), evaluating the determinant and simplifying successively yields

$$
\begin{aligned}
\operatorname{sgn}\left(\frac{\partial \underline{a}}{\partial g}\right) & =\operatorname{sgn}\left(\left|\begin{array}{cc}
-A \pi & A \pi \\
\pi\left[\lambda H^{\prime \prime}(\underline{m})+(1-\lambda) H^{\prime \prime}(\bar{m})\right] & (1-\pi) U^{\prime \prime}(s)+\pi\left[\lambda H^{\prime \prime}(\underline{m})+(1-\lambda) H^{\prime \prime}(\bar{m})\right]
\end{array}\right|\right) \\
& =\operatorname{sgn}\left(-\pi A\left[(1-\pi) u^{\prime \prime}(s)\right]\right)=\operatorname{sgn}(A) .
\end{aligned}
$$


To sum up we have to study the sign of $A$ defined by (A.5). Substituting from (15) and rearranging yields

$$
A=(1-\lambda) \Delta v_{A}\left[H^{\prime \prime}(\underline{m}) \frac{H^{\prime}(\bar{m})}{H^{\prime}(\underline{m})}-H^{\prime \prime}(\bar{m})\right] .
$$

Because $\Delta v_{A}>0$, this expression has the same sign as the term in brackets on the RHS.

Consequently we have

$$
\begin{aligned}
A>0 & \Longleftrightarrow \quad \frac{H^{\prime \prime}(\underline{m})}{H^{\prime}(\underline{m})}>\frac{H^{\prime \prime}(\bar{m})}{H^{\prime}(\bar{m})} \\
& \Longleftrightarrow \quad-\frac{H^{\prime \prime}(\underline{m})}{H^{\prime}(\underline{m})}<-\frac{H^{\prime \prime}(\bar{m})}{H^{\prime}(\bar{m})} .
\end{aligned}
$$

Since $\underline{m}<\bar{m}$ this is true under IARA (Increasing Absolute Risk Aversion), while DARA (Decreasing Absolute Risk Aversion) yields $A<0$.

\section{A.2 Only parents have full information}

If the government does not observe $\beta$, but parents do, the problem of the government is given by

$$
\begin{aligned}
& \max _{\bar{g}, \underline{g}, \bar{\tau}, \underline{\tau}} \quad G^{a f}=w \bar{T}-\pi(\lambda \underline{g}+(1-\lambda) \bar{g})-s+(1-\pi) U(s) \\
& +\pi\left[\lambda H\left(s+a^{f}(\underline{\tau}, \underline{\beta})-\underline{\tau}+\underline{g}\right)+(1-\lambda) H\left(s+a^{f}(\bar{\tau}, \bar{\beta})-\bar{\tau}+\bar{g}\right)\right] \\
& \text { s.t. } \quad a^{f}(\underline{\tau}, \underline{\beta})-\underline{\tau}+\underline{g} \geq a^{f}(\bar{\tau}, \underline{\beta})-\bar{\tau}+\bar{g}, \\
& a^{f}(\bar{\tau}, \bar{\beta})-\bar{\tau}+\bar{g} \geq a^{f}(\underline{\tau}, \bar{\beta})-\underline{\tau}+\underline{g},
\end{aligned}
$$

where we added the relevant $I C$ constraints to (18). We shall assume that (A.6), the constraint from the low-cost type to the high-cost type is binding. This constraint is effectively violated at the full information solution characterized in Section 4.1. To see this recall that this solution implies

$$
a^{f}(\bar{\tau}, \bar{\beta})-\bar{\tau}+\bar{g}=a^{f}(\underline{\tau}, \underline{\beta})-\underline{\tau}+\underline{g}<a^{f}(\underline{\tau}, \bar{\beta})-\underline{\tau}+\underline{g},
$$


so that

$$
\bar{g}<\underline{g}+\bar{\tau}-\underline{\tau}+a^{f}(\underline{\tau}, \bar{\beta})-a^{f}(\bar{\tau}, \bar{\beta}),
$$

which violates condition (A.6). Substituting the incentive constraint into $G^{\text {af }}$ the government's problem can then be rewritten as

$$
\begin{aligned}
\max _{\underline{g}, \bar{\tau}, \underline{\tau}} \quad G^{a f}= & w \bar{T}-\pi\left(\underline{g}+(1-\lambda)\left(\bar{\tau}-\underline{\tau}+a^{f}(\underline{\tau}, \bar{\beta})-a^{f}(\bar{\tau}, \bar{\beta})\right)-s+(1-\pi) U(s)\right. \\
& +\pi \lambda H\left(s+a^{f}(\underline{\tau}, \underline{\beta})-\underline{\tau}+\underline{g}\right)+\pi(1-\lambda) H\left(s+a^{f}(\underline{\tau}, \bar{\beta})-\underline{\tau}+\underline{g}\right) .
\end{aligned}
$$

The FOCs are given by

$$
\begin{aligned}
\frac{\partial G^{a f}}{\partial \underline{g}} & =-\pi+\pi\left[\lambda H^{\prime}(\underline{m})+(1-\lambda) H^{\prime}(\bar{m})\right]=0, \\
\frac{\partial G^{a f}}{\partial \bar{\tau}} & =\pi(1-\lambda)\left[\frac{\partial a^{f}(\bar{\tau}, \bar{\beta})}{\partial \tau}-1\right]=0, \\
\frac{\partial G^{a f}}{\partial \underline{\tau}} & =\pi \lambda H^{\prime}(\underline{m})\left[\frac{\partial a^{f}(\underline{\tau}, \underline{\beta})}{\partial \tau}-1\right]+\pi(1-\lambda)\left(H^{\prime}(\bar{m})-1\right)\left[\frac{\partial a^{f}(\underline{\tau}, \bar{\beta})}{\partial \tau}-1\right]=0 .
\end{aligned}
$$

Recall that $a^{f}(\underline{\tau}, \bar{\beta})>a^{f}(\underline{\tau}, \underline{\beta})$, which implies $\bar{m}=s+a^{f}(\underline{\tau}, \bar{\beta})-\underline{\tau}+\underline{g}>\underline{m}=s+$ $a^{f}(\underline{\tau}, \underline{\beta})-\underline{\tau}+\underline{g}$. Hence, condition (A.7) implies that $H^{\prime}(\bar{m})<1$. Furthermore, using our assumption on $a^{f}$ along with (20), which defines $\underline{\tau}^{f f}$, we have

$$
\frac{\partial a^{f}\left(\underline{\tau}^{f f}, \bar{\beta}\right)}{\partial \tau}>\frac{\partial a^{f}\left(\underline{\tau}^{f f}, \underline{\beta}\right)}{\partial \tau}=1,
$$

so that at $\underline{\tau}^{f f}$ the first term on the RHS of (A.9) is zero while the second term is negative, which in turn implies $\underline{\tau}^{a f}<\underline{\tau}^{f f}$ (from the SOC). This is not surprising. In order to relax the IC constraint, the optimal policy distorts $\underline{\tau}$ downwards, which leads to a downward distortion on $\underline{a}$. Conversely, $\bar{\tau}$ and $\bar{a}$ are not distorted; condition (A.8) is identical to its full information counterpart (19).

Combining (A.7) with (21), the first-order condition for parents' saving, yields

$$
U^{\prime}(s)=\left[\lambda H^{\prime}(\underline{m})+(1-\lambda) H^{\prime}(\bar{m})\right]=1
$$


As in the case with uniform transfers, the optimal LTC policy implies full insurance against dependence but, under asymmetric information, it is not possible to provide insurance against the risk of having high-cost children. 